\title{
The class of algebraically closed p-semilattices is finitely axiomatizable
}

\author{
Jö̈l Adler, Regula Rupp, And Jürg Schmid
}

\begin{abstract}
We prove our title, and thereby establish the base for a positive solution of Albert and Burris' problem on the finite axiomatizability of the model companion of the class of all pseudocomplemented semilattices.
\end{abstract}

\section{Introduction}

The purpose of this paper is to prove its title. It is based on [11] and essentially combines, in a new setting, results of Regula Rupp's PhD Thesis [10] with results of Joel Adler's PhD Thesis [1].

The motivation for this work comes from the problem posed by Albert and Burris in the final paragraph of [3]: "Does the class of pseudo-complemented semilattices have a finitely axiomatizable model companion?"

Together with Adler's 2012 preprint [2], the present paper will provide a positive answer to Albert and Burris' question. In fact, we show here that the class of all algebraically closed pseudocomplemented semilattices - for short: a.c. p-semilattices - is finitely axiomatizable in the first-order language of p-semilattices, by providing four axioms - one of which is distributivitycharacterizing this class.

Recall that the model companion mentioned above consists precisely of all existentially complete - for short, e.c.-p-semilattices and is thus a subclass of the class of all a.c. p-semilattices. Adler's preprint [2] provides finitely many additional axioms singling out the e.c. members within all a.c. p-semilattices, and thus will settle the problem.

The paper is organized as follows. Section 2 collects the basic algebraic notions concerning p-semilattices, while Section 3 provides a short summary of the relevant model-theoretic concepts, adapted to our setting.

In Section 4, we consider distributive meet-semilattices. The main result of the section is that in a distributive p-semilattice $P$, an arbitrary - not necessarily distributive - finite p-subsemilattice $F \unlhd P$ can be extended to a finite distributive p-semilattice $F_{0}$ such that $F \unlhd F_{0} \unlhd P$.

Presented by J. Berman.

Received December 11. 2012; accepted in final form January 28, 2013.

2010 Mathematics Subject Classification: Primary: 03C60; Secondary: 03C05, 03C10, 03G10, 06A12.

Key words and phrases: pseudocomplemented semilattice, algebraic and existential closedness, finite axiomatizability. 
In Section 5, we specify an axiom (A1) guaranteeing that $F_{0}$, as obtained in Section 4, can be extended to a finite distributive p-semilattice $F_{1}$ with $F_{0} \unlhd F_{1} \unlhd P$ such that the dense elements of $F_{1}$ form a boolean meet-semilattice under the induced order.

In Section 6, another axiom, (A2), is introduced, and it is shown that $F_{1}$, as obtained in Section 5, can be extended to a finite p-semilattice $F_{2}$ with $F_{1} \unlhd F_{2} \unlhd P$ such that $F_{2}$ is isomorphic to a direct product of subdirectly irreducible p-semilattices, provided $P$ satisfies (A2).

In Section 7 , it is shown that in a p-semilattice $P$ satisfying an additional axiom (A3), any finite p-subsemilattice $F_{2}$, as obtained in Section 6 , can be extended to a p-subsemilattice $F_{3} \unlhd P$ isomorphic to a direct product with finitely many factors, each of them being either the two-element boolean psemilattice or the unique countable atom-free boolean algebra with a new top element added.

Section 8 establishes the necessity of the above axioms for a p-semilattice to be algebraically closed. Finally, Section 9 formulates our main theorem.

\section{Pseudocomplemented semilattices}

A pseudocomplemented semilattice (for short: p-semilattice) $\left(P ; \wedge,{ }^{*}, 0,1\right)$ is a meet-semilattice $(P ; \wedge)$ with least element 0 and top element 1 , equipped with an unary operation $a \mapsto a^{*}$ such that for all $x \in P, x \wedge a=0$ iff $x \leq a^{*}$. It is a nontrivial fact that the class PCS of all p-semilattices can be (finitely) axiomatized by identities in the first-order language $\mathcal{L}_{P C S}=\left\{\wedge,{ }^{*}, 0,1\right\}$, making PCS a variety; see [5]. We freely write $P$ for the p-semilattice $\left(P ; \wedge,{ }^{*}, 0,1\right)$ (and similarly for algebraic structures in general whenever the operations and relations under consideration are clear from the context). An element $d \in P$ satisfying $d^{*}=0$ is called dense. $\mathrm{D}(P)$ denotes the set of all dense elements of $P$; moreover, $(\mathrm{D}(P) ; \wedge, 1)$ is a subsemilattice - in fact, a filter - of $(P ; \wedge, 1)$. Further, $d \in \mathrm{D}(P)$ is called maximally dense iff $d \neq 1$ and $d \leq d^{\prime} \leq 1$ implies $d^{\prime}=d$ or $d^{\prime}=1$. An element $s \in P$ is called skeletal iff $s^{* *}=s$. The set of all skeletal elements of $P$ is denoted by $\operatorname{Sk}(P)$; it is a subalgebra of the p-semilattice $P$. Within $\operatorname{Sk}(P)$ the supremum of two elements exists w.r.t. to the order inherited from $P$; in fact, $\sup _{\mathrm{Sk}}\{a, b\}=\left(a^{*} \wedge b^{*}\right)^{*}$ for $a, b \in \operatorname{Sk}(P)$. Setting $a \sqcup b=\left(a^{*} \wedge b^{*}\right)^{*},\left(\operatorname{Sk}(P) ; \wedge, \sqcup,{ }^{*}, 0,1\right)$ is a boolean algebra. The set of all atoms of $P$ is denoted by $\operatorname{At}(P)$.

For any p-semilattice $P$, a p-semilattice $\hat{P}$ is obtained from $P$ by adding a new top element. In most cases, the top element of $P$ will be renamed to $e$ and 1 will stand for the new top element. We write $\mathbf{2}$ for the two-element boolean algebra and $\mathbf{A}$ for the unique countable atom-free boolean algebra.

The class of p-semilattices $P$ that are generated (as p-semilattices) by their skeletal and dense elements - that is, $P=\langle\operatorname{Sk}(P) \cup \mathrm{D}(P)\rangle_{\mathbf{P C S}}$-play an important rôle in our context. They are called representable; equivalently, $P$ is representable iff every $x \in P$ admits a (not necessarily unique) representation 
of the the form $x=x^{* *} \wedge d$ for some $d \in \mathrm{D}(P)$. Obviously, in this case, $P=\{b \wedge d: b \in \mathrm{Sk}(P), d \in \mathrm{D}(P)\}$.

Although there is only one binary operation in a p-semilattice $P$, a notion of distributivity can be introduced: Call $P$ distributive if for all $a, b, c \in P$ with $c \geq a \wedge b$, there exist $x, y \in P$ satisfying $x \geq a, y \geq b$, and $x \wedge y=c$. Distributivity in p-semilattices - in particular, its relationship with representability — will be considered in detail in Section 4.

For a p-semilattice $P$ and a skeletal element $a \in P$, the binary relation $x \theta_{a} y: \Longleftrightarrow a \wedge x=a \wedge y$ is a PCS-congruence. The factor algebra $P / \theta_{a}$ is isomorphic to $\left.(\{a \wedge x: x \in P\} ; \cdot)^{\prime}, 0, a\right)$, where $(a \wedge x) \cdot(a \wedge y)$ is defined as $a \wedge(x \wedge y)$ and $(a \wedge x)^{\prime}$ as $a \wedge x^{*}$. Furthermore, the map $f_{a}: P \rightarrow P / \theta_{a}$ defined by $f_{a}(x)=a \wedge x$ is a surjective homomorphism. The following special case will frequently occur: Consider a direct product $P=\prod_{i \in I} P_{i}$ of p-semilattices, and a subset $J \subseteq I$. Then $\prod_{i \in J} P_{i} \cong P / \theta_{a}$, where $a \in P$ is given by $(a)_{i}=1$ iff $i \in J$, and by $(a)_{i}=0$ iff $i \in I \backslash J$.

In a general meet-semilattice $\left(S ; \wedge\right.$ ), $\downarrow_{S} x$ (or simply $\downarrow x$ if $S$ is clear from the context) stands for $\{y \in S: y \leq x\}$, the down-set generated by $x$ in $S$, where $x$ is any element of $S$. We write $\mathcal{O}(S)$ for the (distributive) lattice of all down-sets of $S$ ordered by set inclusion. Note that if $S$ is finite, then $\downarrow x$ is actually a lattice under its induced order for any $x$, and we thus will call $x \in S$ join-irreducible iff $x$ is such in $\downarrow x$, for $S$ finite. We write $\mathcal{J}(F)$ for the set of all (non-zero) join-irreducibles of a finite meet-semilattice $F$. Whenever there is no danger of confusion, $\mathcal{J}(F)$ also stands for the poset of all join-irreducibles under the order inherited from $F$.

Finally, a meet-semilattice $(S ; \wedge)$ is called boolean iff it is the $\wedge$-reduct of a boolean algebra. We use $Q \unlhd P$ (respectively $P \unrhd Q$ ) freely to indicate that $Q$ is a subalgebra of $P$ in whatever signature $P$ and $Q$ are considered at the moment. More background on (p-)semilattices may be found in [5] and [7], or in $[4]$.

\section{Model theory}

For a given p-semilattice $P$, let $\mathcal{L}_{P C S}^{P}$ be the language obtained from $\mathcal{L}_{P C S}$ by adding bijectively a new constant symbol for each $a \in P$ to $\mathcal{L}_{P C S} . P$ is called algebraically closed - abbreviated by a.c.-(in PCS) if $P$ satisfies every positive existential $\mathcal{L}_{P C S^{-}}^{P}$-sentence that holds in some extension $P \unlhd P^{\prime}$ with $P^{\prime} \in$ PCS. In plainer terms, $P$ is a.c. iff every finite system of $P C S$-equations with coefficients from $P$ that is solvable in some extension $P \unlhd P^{\prime} \in$ PCS already has a solution in $P$. The stronger notion of being existentially completenot considered in this paper but crucial in the problem posed by Albert and Burris in [3] - just differs from a.c. by allowing also (finitely many) negated equations; the model companion of PCS mentioned in the introduction is then just the class of all existentially complete algebras in PCS. For more 
background on the model theory relevant here, the reader is referred to [6], especially Chapter 7 . We use $\omega$ to denote the set of all natural numbers.

In [11] the following characterization of algebraically closed p-semilattices is established.

Theorem 3.1. A p-semilattice $P$ is algebraically closed iff for any finite subalgebra $F \unlhd P$, there exists a p-semilattice $F^{\prime}$ isomorphic to $\mathbf{2}^{r} \times(\hat{\mathbf{A}})^{s}$ for some $r, s \in \omega$ such that $F \unlhd F^{\prime} \unlhd P$.

Note that the trivial one-element p-semilattice is a.c., since it only can be embedded into itself. Write A(PCS) for the class of all a.c. members of PCS. The main result of this paper is a finite list of $\mathcal{L}_{P C S}$-sentences that hold in $P \in \mathbf{P C S}$ iff $P$ is a.c.; what actually will be shown is that these sentences hold in $P$ iff $P$ has the extension property specified in Theorem 3.1 above.

The remainder of this section collects some results from [1], providing evidence that a finite axiomatization of $\mathbf{A}(\mathbf{P C S})$ should exist. So far, the only members of $\mathbf{A}(\mathbf{P C S})$ identified immediately by Theorem 3.1 are the direct products $\mathbf{2}^{r} \times(\hat{\mathbf{A}})^{s}$ for some $r, s \in \omega$. There are others:

Let $Q$ be the subalgebra of $(\hat{\mathbf{A}})^{\omega}$ jointly generated by $\operatorname{Sk}\left((\hat{\mathbf{A}})^{\omega}\right)$ and $D_{e}:=$ $\left\{d \in \mathrm{D}\left((\hat{\mathbf{A}})^{\omega}\right):(d)_{i}=e\right.$ for at most finitely many $\left.i \in \omega\right\}$.

It is easy to see that $Q=\left\{a \wedge d: a \in \operatorname{Sk}\left((\hat{\mathbf{A}})^{\omega}\right)\right.$ and $\left.d \in \mathrm{D}_{e}\right\}$, since the latter set evidently is closed under $\wedge$ and $(a \wedge d)^{* *}=a^{* *} \wedge d^{* *}=a^{* *} \wedge 1=$ $a^{* *} \in \operatorname{Sk}\left((\hat{\mathbf{A}})^{\omega}\right)$.

Note that $Q$ is not isomorphic to any direct product with factors $\mathbf{2}$ or $\hat{B}(B$ any boolean algebra), since such a product has either a finite or uncountable number of dense elements while $\mathrm{D}(Q)=D_{e}$ is countable.

Let $F \unlhd Q$ be finite. There exists a least $n_{F} \in \omega$ such that $(x)_{i} \neq e$ for all $x \in F$ and $i>n_{F}$. Define an element $a \in Q$ by $(a)_{i}=1$ for $i \leq n_{F}$ and $(a)_{i}=0$ for $i>n_{F}$. Let $Q_{a}=Q / \theta_{a}$ (see Section 2) and $F_{a}=F / \theta_{a} \cap(F \times F)$; define $Q_{a^{*}}$ and $F_{a^{*}}$ analogously. Now $Q \cong Q_{a} \times Q_{a^{*}}$ canonically, $F_{a} \unlhd Q_{a}$, $F_{a^{*}} \unlhd Q_{a^{*}}$, and thus $F \cong F^{\prime} \unlhd F_{a} \times F_{a^{*}}$ for some copy $F^{\prime}$ of $F$. It is clear that $Q_{a} \cong(\hat{\mathbf{A}})^{n}$; moreover, $F_{a^{*}}$ is a finite boolean subalgebra of $\operatorname{Sk}\left(Q_{a^{*}}\right)$, and thus $F_{a^{*}} \cong \mathbf{2}^{k}$ for some $k \in \omega$. Hence, $F \cong F^{\prime} \unlhd F_{a} \times F_{a^{*}} \unlhd(\hat{\mathbf{A}})^{n} \times F_{a^{*}} \unlhd Q_{a} \times Q_{a^{*}}$. Under the canonical isomorphism $Q \cong Q_{a} \times Q_{a^{*}}$, the algebra $(\hat{\mathbf{A}})^{n} \times F_{a^{*}}$ corresponds to a subalgebra of $Q$ of the form required by Theorem 3.1.

That the class of all existentially complete p-semilattices - alias the model companion of PCS - can be axiomatized by $\mathcal{L}_{P C S}$-sentences follows from general model-theoretic properties of PCS, viz., the fact that PCS is a finitely generated universal Horn class with both the amalgamation and joint embedding properties; see [3] for details. No such general argument seems to apply to the (wider) class $\mathbf{A}(\mathbf{P C S})$. In fact, the mere axiomatizability of $\mathbf{A}(\mathbf{P C S})$ was first established in [1].

Now, an axiomatizable class of $\mathcal{L}_{P C S}$-structures is finitely axiomatizable iff both the class itself as well as its complementary class are closed under elementary equivalence and ultraproducts. So partial evidence for the finite 
axiomatizability of $\mathbf{A}(\mathbf{P C S})$ is provided by [1, Theorem 4.1], which states that an ultraproduct of finite p-semilattices that are not a.c. cannot be a.c. either.

\section{Distributivity}

There is a natural notion of distributivity for meet-semilattices, see Subsection 4.2 below. Generally, a subsemilattice of a distributive meet-semilattice need not be distributive. However, a finite subsemilattice $F \unlhd S$ of any distributive meet-semilattice $S$ can always be expanded to a finite distributive meet-semilattice $F^{\prime}$ such that $F \unlhd F^{\prime} \unlhd S$ - a very crucial fact in our context, as we shall see. This fact is well known; to the best of our knowledge, it appeared first in print as Fact 4 in [9]. What we actually need is a p-semilattice version of this result, which does not follow immediately from [9]. Therefore, we present an exposition based on so-called minimal boolean extensions; moreover, the specific properties of such extensions will be crucial in Section 5 when they are used to construct successive distributive extensions by destroying comparabilities between join-irreducibles.

4.1. Minimal boolean extensions. Every semilattice $(S ; \wedge)$ embeds - as a meet-semilattice - into a boolean algebra: Indeed, the map $x \longmapsto \downarrow x$ embeds $S$ into the power set algebra $\mathcal{P}(S)$. If $S$ is finite, so is $\mathcal{P}(S)$, and there exists, therefore, a uniquely determined - up to isomorphism - smallest boolean algebra containing $S$ as a meet-subsemilattice, denoted by $B_{S}$ in the sequel.

So let $(F ; \wedge)$ be an arbitrary but fixed finite meet-semilattice, and put $\operatorname{At}\left(B_{F}\right)=\left\{q_{1}, \ldots, q_{n}\right\}$, thus $B_{F} \cong \mathcal{P}\left\{q_{1}, \ldots, q_{n}\right\}$. We identify $B_{F}$ with its canonical copy $\mathcal{P}\left\{q_{1}, \ldots, q_{n}\right\}$ in the sequel, and fix an embedding $e_{F}: F \rightarrow B_{F}$.

Given $q_{i} \in \operatorname{At}\left(B_{F}\right)$, define $y_{i} \in F$ by $y_{i}=\bigwedge\left\{x \in F: q_{i} \in e_{F}(x)\right\}$. The doubleton $J_{i}:=\left\{\emptyset,\left\{q_{i}\right\}\right\}$ is a nontrivial ideal in $B_{F}$, so $F$ will no longer embed into $B_{F} / J_{i}$. With $p: B_{F} \rightarrow B_{F} / J_{i}$ the canonical (boolean) epimorphism, we thus find $u \neq v \in F$ such that $\left(p \circ e_{F}\right)(u)=\left(p \circ e_{F}\right)(v)$. It follows that, say, (i) $q_{i} \in e_{F}(u)$ but (ii) $q_{i} \notin e_{F}(v)$. We infer (i) $u \geq y_{i}$ and (ii) $v \not y_{i}$, so $y_{i}=u \wedge y_{i}>u \wedge y_{i} \wedge v=y_{i} \wedge v$. But

$$
\begin{aligned}
\left(p \circ e_{F}\right)\left(y_{i}\right) & =\left(p \circ e_{F}\right)\left(y_{i}\right) \cap\left(p \circ e_{F}\right)(u) \\
& =\left(p \circ e_{F}\right)\left(y_{i}\right) \cap\left(p \circ e_{F}\right)(v)=\left(p \circ e_{F}\right)\left(y_{i} \wedge v\right),
\end{aligned}
$$

and we conclude that the sets $e_{F}\left(y_{i}\right)$ and $e_{F}\left(y_{i} \wedge v\right)$ differ exactly in the point $q_{i}$. In other words, $y_{i}$ has $y_{i} \wedge v$ as its unique lower neighbor in $F$, that is, $y_{i} \in \mathcal{J}(F)$.

Conversely, consider $y \in F$ join-irreducible with lower neighbor $y^{\prime}$. Suppose we find atoms $q_{i} \neq q_{j}$ in $e_{F}(y) \backslash e_{F}\left(y^{\prime}\right)$. Put $J=\left\{\emptyset,\left\{q_{j}\right\}\right\}, J$ is a nontrivial ideal in $B_{F}$. With $p: B_{F} \rightarrow B_{F} / J$ the canonical epimorphism, it follows that $p \circ e_{F}$ is a monomorphism, contradicting the minimality of $B_{F}$. This shows that $e_{F}(y) \backslash e_{F}\left(y^{\prime}\right)$ must be a singleton. 
Summing up, we have established a bijective correspondence between $\mathcal{J}(F)$ and $\operatorname{At}\left(B_{F}\right)$, and we will identify the two sets in the sequel. This means that $B_{F}$ is taken to be the powerset algebra $\mathcal{P}(\mathcal{J}(F))$. Next, define $\mathrm{O}_{F}: F \rightarrow$ $\mathcal{P}(\mathcal{J}(F))$ by $\mathrm{O}_{F}(x)=\downarrow x \cap \mathcal{J}(F)$. Since $x=\sup _{\downarrow x} \mathrm{O}_{F}(x)$, we see that $\mathrm{O}_{F}$ is injective. Also, since $y \leq x_{1} \wedge x_{2}$ iff $y \leq x_{1}$ and $y \leq x_{2}$ for $x_{1}, x_{2} \in F$, and as $y \in \mathcal{J}(F)$, we have $\mathrm{O}_{F}\left(x_{1} \wedge x_{2}\right)=\mathrm{O}_{F}\left(x_{1}\right) \cap \mathrm{O}_{F}\left(x_{2}\right)$ for all $x_{1}, x_{2} \in F$. So, $\mathrm{O}_{F}$ actually is an embedding of $F$ into $\mathcal{P}(\mathcal{J}(F))$.

Definition 4.1. The pair $\left(\mathcal{P}(\mathcal{J}(F)), \mathrm{O}_{F}\right)=\left(B_{F}, \mathrm{O}_{F}\right)$ is the (canonical) minimal boolean extension of a finite meet-semilattice $F$.

For easier reference, we also write $\hat{x}$ instead of $\downarrow x \cap \mathcal{J}(F)=\mathrm{O}_{F}(x)$ for elements $x$ of finite meet-semilattices $F$ (there is no danger of confusion with the notation $\hat{P}$ introduced for p-semilattices in Section 2).

4.2. Distributivity in meet-semilattices. The canonical notion of distributivity for meet-semilattices is captured by:

Definition 4.2. A meet-semilattice $S$ is distributive iff for all $a, b, c$ in $S$, the following holds: Whenever $c \geq a \wedge b$, there exist $x, y \in S$ such that $x \geq a$, $y \geq b$, and $x \wedge y=c$.

It is clear that this property can be expressed by a sentence (DIST) in (the $\wedge$-reduct of) $\mathcal{L}_{P C S}$.

The above definition of distributivity in meet-semilattices is closely related to distributivity in lattices:

Remark 4.3. For any lattice $(L ; \wedge, \vee)$, its meet-semilattice reduct $(L ; \wedge)$ satisfies (DIST) iff $L$ is distributive as a lattice. Alternatively, $(S ; \wedge)$ is distributive as a meet-semilattice iff the poset of all nonempty filters of $S$, ordered by set inclusion, is a distributive lattice.

Note that distributivity in meet-semilattices is not necessarily inherited by subsemilattices: Let $\mathbf{2}$ be the 2 -element chain $0<1$. Then $\mathbf{2} \times \mathbf{2} \backslash\{(1,1)\}$ is a nondistributive meet-subsemilattice of the distributive lattice $\mathbf{2} \times \mathbf{2}$.

Lemma 4.4. A distributive p-semilattice is representable.

Proof. Obviously, $x \geq 0=x^{* *} \wedge x^{*}$. Using distributivity we find $a, b \in P$ such that $a \geq x^{* *}, b \geq x^{*}$ and $a \wedge b=x$. Meeting both sides of the last equation with $x^{* *}$ we obtain $a \wedge x^{* *} \wedge b=x \wedge x^{* *}$, that is, $x^{* *} \wedge b=x$. But $b \in \mathrm{D}(P)$, since $b \geq x, x^{*}$ and thus $b^{*} \leq x^{*}, x^{* *}$, that is, $b \leq 0=x^{*} \wedge x^{* *}$.

The converse of Lemma 4.4 does not hold as easy examples show. However, the distributivity of a representable p-semilattice depends only on its dense elements, as we will show presently.

For the purpose of this paper, call an element $x$ of an arbitrary p-semilattice $P$ distributive iff for any $a, b \in P, x \geq a \wedge b$ implies the existence of $x_{a}, x_{b} \in P$ such that $x_{a} \geq a, x_{b} \geq b$ and $x_{a} \wedge x_{b}=x$. It is routine to check that the meet of two distributive elements is distributive in any p-semilattice. 
Lemma 4.5. A representable $p$-semilattice $P$ is distributive iff every $d \in \mathrm{D}(P)$ is distributive.

Proof. Note first that skeletal elements are distributive in any p-semilattice: Indeed, consider $a, b \in P$ and $c \in \operatorname{Sk}(P)$ such that $c \geq a \wedge b$. This implies $c=c^{* *} \geq a^{* *} \wedge b^{* *}$. By boolean distributivity, we obtain $\left(c \sqcup a^{* *}\right) \wedge\left(c \sqcup b^{* *}\right)=$ $c \sqcup\left(a^{* *} \wedge b^{* *}\right)=c$, with $c \vee a^{* *} \geq a^{* *} \geq a$ and $c \vee b^{* *} \geq b^{* *} \geq b$. Since $P$ is representable, we have $x=x^{* *} \wedge d_{x}$ with suitable $d_{x} \in \mathrm{D}(P)$ for any $x \in P$. So $x$ as the meet of two distributive elements is distributive provided every $d \in \mathrm{D}(P)$ is such.

Given a distributive meet-semilattice $S$ and a subsemilattice $F \unlhd S$, it is trivial to find a distributive semilattice $F^{\prime}$ such that $F \unlhd F^{\prime} \unlhd S$ : Just take $F^{\prime}=S$. It turns out to be less trivial to find, for $F$ finite, a finite distributive $F^{\prime}$ extending $F$ within $S$. Proposition 4.9 asserts that this is always possible. Moreover, in Proposition 4.11, we will show that the same is true within the class of all pseudocomplemented meet-semilattices.

Lemma 4.6. Let $S$ be a distributive meet-semilattice, and let $a, a_{1}, \ldots, a_{n}, b, c$ be elements of $S$.

(i) If $a \wedge b \leq c \leq b$, there exists $x \in S$ such that $x \geq a$ and $x \wedge b=c$.

(ii) If $a_{1} \wedge c=\cdots=a_{n} \wedge c$, there exists $x \in S$ such that $x \geq a_{i}(1 \leq i \leq n)$ and $x \wedge c=a_{1} \wedge c$.

Proof. (i): Let $a \wedge b \leq c \leq b$. Using distributivity, we find $x, y \in S$ with $x \geq a, y \geq b$, and $x \wedge y=c$. Since $b \geq c$, we obtain $c=x \wedge y=x \wedge y \wedge b=x \wedge b$.

(ii): Suppose $a_{1} \wedge c=\cdots=a_{n} \wedge c$ and consider $a_{1} \wedge c=a_{2} \wedge c$. Using distributivity on $a_{1} \geq a_{2} \wedge c$, find $u_{2}, u_{c} \in S$ such that $u_{2} \geq a_{2}, u_{c} \geq c$, and $u_{2} \wedge u_{c}=a_{1}$ (thus, $\left.u_{2} \geq a_{1}, a_{2}\right)$. Analogously, $a_{2} \geq a_{1} \wedge c$ gives the existence of $v_{1}, v_{c} \in S$ satisfying $v_{1} \geq a_{1}, v_{c} \geq c$, and $v_{1} \wedge v_{c}=a_{2}$ (thus, $v_{1} \geq a_{1}, a_{2}$ ). Put $x_{12}=v_{1} \wedge u_{2}$; then $x_{12} \geq a_{1}, a_{2}$. Moreover,

$$
\begin{aligned}
x_{12} \wedge c & =x_{12} \wedge u_{c} \wedge v_{c} \wedge c=v_{1} \wedge u_{2} \wedge u_{c} \wedge v_{c} \wedge c \\
& =a_{1} \wedge a_{2} \wedge c=a_{1} \wedge c=a_{c} \wedge c .
\end{aligned}
$$

Repeat this process suitably often, first proceeding with $x_{12} \wedge c=a_{3} \wedge c=$ $\cdots=a_{n} \wedge c$.

Corollary 4.7. A distributive meet-semilattice $S$ is upwards directed, that is, any two elements have a common upper bound in $S$. If $S$ is also finite, then it is a distributive lattice under its natural order.

Proof. Putting $c=b$ in Lemma 4.6(i), obtain $x$ as a common upper bound for $a$ and $b$. If $S$ is finite, it will thus contain a greatest element, and thus the supremum of any two elements.

Going back to the minimal boolean extension $B_{F}$ of a finite meet-semilattice $F$, note that $\mathrm{O}_{F}(x)$ is actually a down-set in $\mathcal{J}(F)$. Hence, $\mathrm{O}_{F}$ embeds 
$F$ into the sublattice $\mathcal{O}(\mathcal{J}(F))$ of $\mathcal{P}(\mathcal{J}(F))$. For easier reference, we also write $L_{F}$ for the distributive lattice $\mathcal{O}(\mathcal{J}(F)) ; L_{F}$ is generated, as a lattice, by $\left\{\mathrm{O}_{F}(y): y \in \mathcal{J}(F)\right\}$ and is (up to isomorphism) the uniquely determined minimal distributive lattice embedding $F$. By Corollary $4.7, L_{F}$ can also be characterized as the (unique up to isomorphism) minimal distributive meetsemilattice embedding $F$. Note also that $B_{L_{F}} \cong B_{F}$ canonically.

Corollary 4.8. For any finite meet-semilattice $F, \mathrm{O}_{F}$ provides an embedding of $F$ into $L_{F}$. Moreover, $F$ is distributive iff $\mathrm{O}_{F}$ is an isomorphism between $F$ and $L_{F}$.

4.3. Distributive extensions. The basic result, for our purposes, is:

Proposition 4.9. Assume $S$ is a distributive meet-semilattice and $F \unlhd S$ a finite subsemilattice of $S$. Then there exists a finite distributive semilattice $F_{0}$ such that $F \unlhd F_{0} \unlhd S$. In fact, we find such $F_{0}$ satisfying $F_{0} \cong L_{F}$.

Proof. Obviously, $F$ has a least element $0_{F}$, since it is finite. Moreover, we can assume without loss of generality that $F$ has a greatest element $1_{F}$ : If not, there is an upper bound $s$ for $F$ within $S$ by Corollary 4.7 as $S$ is distributive. Clearly, $F_{s}:=F \cup\{s\}$ is a subsemilattice of $S$ extending $F$, and we can proceed by replacing $F$ by $F_{s}$.

Suppose $F$ is not distributive. Then the embedding $\mathrm{O}_{F}: F \rightarrow L_{F}$ cannot be surjective by Corollary 4.8, so there exists a down-set $H \subseteq \mathcal{J}(F)$ such that $H \notin i m \mathrm{O}_{F}$. Pick $H_{0}$ minimal with this property. This means that $H_{0} \neq \hat{w}$ for any $w \in F$, but $H=\widehat{w_{H}}$ for a unique $w_{H}$ whenever $H$ is a down-set in $\mathcal{J}(F)$ strictly contained in $H_{0}$. Let $\left\{j_{1}, \ldots, j_{r}\right\}$ be the complete list of all maximal elements in $H_{0}$. It follows that $r \geq 2$ for otherwise $H_{0}=\widehat{j_{1}}$. Put $u=\sup _{F} H_{0}=\sup _{F}\left\{j_{1}, \ldots, j_{r}\right\}$, which exists, since $F$ has a greatest element. Note that $u \notin \mathcal{J}(F): u \neq j_{k}$ for all $1 \leq k \leq r$, since $r \geq 2$, so if $u \in \mathcal{J}(F)$ with lower cover $u^{-}$, then $u^{-} \geq j_{k}$ for all $1 \leq k \leq r$, contradicting $u=\sup _{F} H_{0}$.

Let $U=\left\{x \in F: \hat{x} \supseteq H_{0}\right\}, L=\left\{x \in F: \hat{x} \subseteq H_{0}\right\}$, and $I=F \backslash(U \cup L)$. Note that $U$ is a nonempty up-set in $F\left(1_{F} \in U\right), L$ is nonempty down-set in $F\left(0_{F} \in L\right)$, and $L \cap U=\emptyset$ by the choice of $H_{0}$. Also, $I \neq \emptyset$, since otherwise $H_{0}=\hat{u}$. Pick $x \in I$. It follows that $\hat{x} \cap H_{0} \subset H_{0}$, and thus $\hat{x} \cap H_{0}=\widehat{w_{x}}$ for some $w_{x} \in L$. So $w_{x} \neq x$ and $\widehat{w_{x}} \subseteq \hat{x}$, which implies $w_{x}<x$, since is an embedding of $F$ into $L_{F}$ by Corollary 4.8. Further, consider $j_{k}$ with $1 \leq k \leq r$. Since $\widehat{j_{k}} \subseteq H_{0}$, we have $\hat{x} \cap \widehat{j_{k}} \subseteq \hat{x} \cap H_{0}=\widehat{w_{x}}$. Invoking the embedding property of ^ again, we conclude that $x \wedge j_{k} \leq w_{x}$.

Using distributivity of $S$ and Lemma 4.6(i), we find, for $1 \leq k \leq r$, an element $a_{k} \in S$ satisfying $a_{k} \geq j_{k}$ and $a_{k} \wedge x=w_{x}<x$. By Lemma 4.6(ii), we then find $b_{x} \in S$ satisfying $b_{x} \geq a_{k}\left(\geq j_{k}\right)$ for $1 \leq k \leq r$ and $b_{x} \wedge x=w_{x}$; moreover, $b_{x} \ngtr x$ for otherwise $w_{x}=b_{x} \wedge x=x$.

Define $b \in S$ by $b=\bigwedge_{x \in I} b_{x} \wedge u$. We claim that for any $y \in F$, either $b \wedge y=b$ or $b \wedge y \in F$ : If $y \in U$, then $y \geq u \geq b$, and thus $b \wedge y=b$. If $y \in L$, then also $b \wedge y \in L$, since $L$ is a down-set in $F$. If $y \in I$, then 
$b \wedge y \leq b_{y} \wedge y=w_{y} \in L$, and thus $b \wedge y \in L$ again. It follows that $F_{b}:=F \cup\{b\}$ is a subsemilattice of $S$ containing $F$.

Consider $j \in \mathcal{J}(F)$ such that $j \leq b$ : If $j \in U$, then $j \geq u$, in fact, $j>u$, since $u \notin \mathcal{J}(F)$. Hence, $j \not \leq b \leq u$. If $j \in L$, then $j \leq u$ and $j \leq j_{k}$ for some $1 \leq k \leq r$, which implies $j \leq b_{x}$ for all $x \in I$, thus $j \leq b$. If $j \in I$, then $j \not \leq b_{j}$ as shown above, thus $j \not \leq b$. Summing up, the downset induced by $b$ in $\mathcal{J}(F)$ is $L \cap \mathcal{J}(F)=H_{0}$. Moreover, $\mathrm{O}_{F} \cup\left\{\left(b, H_{0}\right)\right\}$ obviously is the canonical isomorphism $\mathrm{O}_{F_{b}}$ between $F_{b}$ and the subsemilattice generated within $L_{F}=\mathcal{O}(\mathcal{J}(F))$ by $\operatorname{imO}_{F} \cup\left\{H_{0}\right\}$. Repeat the procedure with $F_{b}$ and iterate; the process breaks off with an isomorphism between some subsemilattice $F_{0}$ of $S$ and $L_{F}$, making $F_{0}$ distributive.

Since our concern is with p-semilattices, the natural question is whether any finite p-subsemilattice $F$ of a distributive p-semilattice $P$ can be extended, within $P$, to a finite distributive p-subsemilattice $F^{\prime}$ of $P$.

The starting observation is that $L_{F}=\mathcal{O}(\mathcal{J}(F))$, as a finite distributive lattice, is pseudocomplemented for any meet-semilattice $F$. Indeed, for any down-set $H \subseteq \mathcal{J}(F)$ the set $H^{+}:=\{j \in \mathcal{J}(F): \hat{\jmath} \cap H=\emptyset\}$ is its pseudocomplement.

Lemma 4.10. Let $F$ be a finite p-semilattice. Then the canonical embedding $\mathrm{O}_{F}: F \rightarrow L_{F}$ preserves pseudocomplements.

Proof. We have to show that $\widehat{x^{*}}=\hat{x}^{+}$for all $x \in F$. Now, $j \leq x^{*}$ iff $j \wedge x=0$ for all $j \in \mathcal{J}(F)$, and thus

$$
\begin{aligned}
\hat{x}^{+} & =\{j \in \mathcal{J}(F): \hat{\jmath} \cap \hat{x}=\emptyset\}=\{j \in \mathcal{J}(F): j \wedge x=0\} \\
& =\left\{j \in \mathcal{J}(F): j \leq x^{*}\right\}=\widehat{x^{*}} .
\end{aligned}
$$

This is enough to prove the following result.

Corollary 4.11. Assume $P$ is a distributive p-semilattice and $F$ a finite $p$-subsemilattice of $P$. Then there exists a finite distributive $p$-semilattice $F_{0}$ such that $F \unlhd F_{0} \unlhd P$. In fact, we find such $F_{0}$ satisfying $F_{0} \cong L_{F}$.

Proof. Consider $F_{b}$ as in the proof of Proposition4.9 (note that $1_{F}$ exists and equals $\left.1_{P}\right)$. All we need to show is that $F_{b}$ is closed under pseudocomplements and that $\mathrm{O}_{F} \cup\left\{\left(b, H_{0}\right)\right\}$ preserves pseudocomplements. We claim that $b^{*}=u^{*}$, and thus $b^{*} \in F \subseteq F_{b}$. Indeed, since $b \leq u$, we have (1): $b^{*} \geq u^{*}$. Further, $j_{k} \leq b(1 \leq k \leq r)$, thus $j_{k}^{*} \geq b^{*}$, and so $(2): j_{1}^{*} \wedge \cdots \wedge j_{r}^{*} \geq b^{*}$. Also, $j_{1}^{*} \wedge \cdots \wedge j_{r}^{*} \leq j_{k}^{*}(1 \leq k \leq r)$, hence $\left(j_{1}^{*} \wedge \cdots \wedge j_{r}^{*}\right)^{*} \geq j_{k}^{* *} \geq j_{k}$ for all $k$, which implies $\left(j_{1}^{*} \wedge \cdots \wedge j_{r}^{*}\right)^{*} \geq u$, and finally $(3):\left(j_{1}^{*} \wedge \cdots \wedge j_{r}^{*}\right)^{* *} \leq u^{*}$. But certainly, (4): $j_{1}^{*} \wedge \cdots \wedge j_{r}^{*} \leq\left(j_{1}^{*} \wedge \cdots \wedge j_{r}^{*}\right)^{* *}$. Putting all together, we obtain

$$
j_{1}^{*} \wedge \cdots \wedge j_{r}^{*} \leq\left(j_{1}^{*} \wedge \cdots \wedge j_{r}^{*}\right)^{* *} \leq u^{*} \leq b^{*} \leq j_{1}^{*} \wedge \cdots \wedge j_{r}^{*},
$$

using (4), (3), (1), and (2), respectively; this proves our claim. Finally, that $\mathrm{O}_{F} \cup\left\{\left(b, H_{0}\right)\right\}$ preserves pseudocomplements is immediate. 


\section{Making the dense filter boolean}

Suppose $F_{0}$ is a finite distributive p-subsemilattice of a distributive psemilattice $P$. The purpose of this section is to show that we can find, provided $P$ satisfies a certain condition (A1), a finite distributive p-subsemilattice $F_{1} \unlhd P$ such that $F_{0} \unlhd F_{1} \unlhd P$ and the dense filter $\mathrm{D}\left(F_{1}\right)$ is boolean.

We start by characterizing finite distributive p-semilattices $F$ with boolean dense filters $\mathrm{D}(F)$ in terms of their associated posets $\mathcal{J}(F)$. Write $\mathcal{J}(F)_{\text {min }}$ for the set of all minimal elements of $\mathcal{J}(F)$.

Lemma 5.1. The dense filter of a finite distributive $p$-semilattice $F$ is boolean iff $\mathcal{J}(F) \backslash \mathcal{J}(F)_{\min }$ is an antichain.

Proof. Using $F \cong L_{F} \cong \mathcal{O}(\mathcal{J}(F))$, it is immediate that $\mathcal{J}(F)_{\text {min }}$ represents the unique minimal dense element of $F$, and that $\mathrm{D}(F)$ is isomorphic to the collection of all down-sets $H \subseteq \mathcal{J}(F)$ containing $\mathcal{J}(F)_{\text {min }}$ (under set inclusion), which in turn is isomorphic to the collection of all down-sets in $\mathcal{J}(F) \backslash \mathcal{J}(F)_{\text {min }}$ (again under set inclusion). But for any finite poset $Q$, one has that $\mathcal{O}(Q)$ is boolean iff $Q$ is an antichain.

Consider any poset $Q$ with order relation $\leq$ and $a, b \in Q$ such that $b$ covers $a$ w.r.t. to $\leq$. It is easy to see that $\leq^{\prime}:=\leq \backslash\{(a, b)\}$ is also an order relation on $Q$ : Dropping $(a, b)$ from $\leq$ does neither affect reflexivity nor antisymmetry, and since $b$ covers $a$ w.r.t. $\leq,(a, b)$ cannot be forced back into $\leq^{\prime}$ by applying transitivity to $\leq^{\prime}$. We will use the short-hand notation $Q_{a b}^{\prime}$ for the resulting poset $\left(Q ; \leq^{\prime}\right)$. We want to describe $\mathcal{O}\left(Q_{a b}^{\prime}\right)$ :

Lemma 5.2. Consider $Q, a, b$ as above and let $M$ the uniquely determined maximal down-set in $\mathcal{O}(Q)$ not containing a. Then

$$
\mathcal{O}\left(Q_{a b}^{\prime}\right)=\mathcal{O}(Q) \cup\{U \cup\{b\}: U \in \mathcal{O}(Q) \text { and } \downarrow b \cap M \subseteq U \subseteq M\} .
$$

Proof. Since $\mathcal{O}\left(Q_{a b}^{\prime}\right) \supseteq \mathcal{O}(Q)$ is clear, a down-set $V \in \mathcal{O}\left(Q_{a b}^{\prime}\right) \backslash \mathcal{O}(Q)$ must contain $b$ but not $a$. If $x \in V \backslash\{b\}$, then $x \in M$, for otherwise, $x \geq a$, putting $a$ in $V$. Moreover, $b>^{\prime} x$ implies $b>x$, and thus $x \in V$, so $V \backslash\{b\} \supseteq \downarrow b \cap M$.

The property (A1) of p-semilattices is defined as follows:

$$
\begin{aligned}
& \left(\forall d_{0}, d_{1}, d_{2} \in \mathrm{D}(P), t \in P\right)(\exists x \in P) \\
& \left(\left(d_{0}<d_{1}<d_{2} \& t \wedge d_{0}<t \wedge d_{1}<t \wedge d_{2}\right) \Longrightarrow\right. \\
& \left.\left(d_{0}<x<d_{2} \& x \wedge d_{1}=d_{0} \& t \wedge d_{0}<t \wedge x<t \wedge d_{2}\right)\right) .
\end{aligned}
$$

Our present aim is to prove

Lemma 5.3. Let $P$ be a distributive $p$-semilattice satisfying (A1) and $F \unlhd P$ a finite distributive $p$-subsemilattice of $P$. Let there be $j_{1}, j_{2} \in \mathcal{J}(F) \backslash \mathcal{J}(F)_{\text {min }}$ such that $j_{2}$ covers $j_{1}$. Then there exists a finite distributive p-semilattice $F^{\prime}$ satisfying $F \unlhd F^{\prime} \unlhd P$ such that $\mathcal{J}\left(F^{\prime}\right)$ is order-isomorphic to $\mathcal{J}(F)_{j_{1} j_{2}}^{\prime}$. 
Proof. Let $H_{0}$ be the unique maximal down-set in $\mathcal{J}(F)$ not containing $j_{1}$. Certainly, $H_{0} \supseteq \mathcal{J}(F)_{\min }$, so $H_{0}$ corresponds to an element $d_{0} \in \mathrm{D}(F)$ under the canonical isomorphism $\mathrm{O}_{F}: F \rightarrow L_{F}$, that is, $\widehat{d_{0}}=H_{0}$. It follows that $w \nsupseteq j_{1}$ iff $w \leq d_{0}$, for any $w \in F$. Note that if $j \in \mathcal{J}(F)$ and $j<j_{2}$, then either $j=j_{1}$ or $j \leq d_{0}$ ( since $j_{2}$ covers $j_{1}$ in $\mathcal{J}(F)$ ). Hence, there are $d_{1}, d_{2} \in \mathrm{D}(F)$ such that $\widehat{d_{1}}=H_{0} \cup\left\{j_{1}\right\}$ and $\widehat{d_{2}}=H_{0} \cup\left\{j_{1}, j_{2}\right\}$.

We conclude that (i) $d_{0}<d_{1}<d_{2}$ and (ii) $j_{2} \wedge d_{0}<j_{2} \wedge d_{1}<j_{2} \wedge d_{2}=j_{2}$. Using (A1) with $t=j_{2}$, we thus find $x \in P$ such that $d_{0}<x<d_{2}, d_{1} \wedge x=d_{0}$, and $j_{2} \wedge d_{0}<j_{2} \wedge x<j_{2} \wedge d_{2}$. Note that $x \in \mathrm{D}(P)$, since $x>d_{0} \in \mathrm{D}(F) \subseteq$ $\mathrm{D}(P)$.

Let $F^{\prime}=\langle F \cup\{x\}\rangle_{P}$ be the p-subsemilattice of $P$ generated by $F$ and $x$. Now, $F \cup\{w \wedge x: w \in F\} \subseteq P$ is obviously closed under meets; moreover, $(w \wedge x)^{*}=(w \wedge x)^{* * *}=\left(w^{* *} \wedge x^{* *}\right)^{*}=\left(w^{* *} \wedge 1\right)^{*}=w^{* * *}=w^{*} \in F$ as $x \in \mathrm{D}(P)$. We conclude that $F^{\prime}=F \cup\{w \wedge x: w \in F\}$.

We analyze the structure of $F^{\prime} \backslash F$ : Suppose $w \in F$ but $w \wedge x \notin F$. Since $x \leq d_{2}$, we have $w \wedge x=w \wedge d_{2} \wedge x$, hence-replacing $w$ by $w \wedge d_{2}$-we can assume without loss of generality that $w \leq d_{2}$. So let $w \leq d_{2}$ and assume, towards a contradiction, that $w \leq d_{1}$. Then $w \wedge x \leq d_{1} \wedge x=d_{0}$, and thus $w \wedge x=w \wedge x \wedge d_{0}=w \wedge d_{0} \in F$, contradicting $w \wedge x \notin F$. So we can assume without loss of generality that $w \not \leq d_{1}$. But, working in $L_{F_{0}}, \hat{w} \subseteq \widehat{d_{2}}$ and $\hat{w} \nsubseteq \widehat{d_{1}}$ are equivalent to $j_{2} \in \hat{w}$, which translates into $j_{2} \leq w$. Summing up, $F^{\prime} \backslash F \subseteq\left\{w \wedge x: j_{2} \leq w \leq d_{2}\right\}$.

Conversely, let $w \in F, j_{2} \leq w \leq d_{2}$, and suppose $w \wedge x \in F$. Then also $j_{2} \wedge w \wedge x=j_{2} \wedge x \in F\left(\right.$ as $\left.j_{2} \leq w\right)$. Now, $j_{2} \wedge d_{0}<j_{2} \wedge x<j_{2} \wedge d_{2}=j_{2}$ by (A1). Looking at $L_{F}$, it is immediate that the unique down-set in $\mathcal{J}(F)$ situated strictly between $\widehat{\jmath_{2}} \cap \widehat{d_{0}}$ and $\widehat{\jmath_{2}}$ is given by $\left(\widehat{\jmath_{2}} \cap \widehat{d_{0}}\right) \cup \widehat{\jmath_{1}}$, and thus contains $j_{1}$. Translated back to $F$, this means that $j_{1} \leq j_{2} \wedge x$, and thus $j_{1} \leq x$. But also $j_{1} \leq d_{1}$ by construction of $d_{1}$, so $j_{1} \leq x \wedge d_{1}=d_{0}$, contradicting the choice of $d_{0}$. We conclude that $w \wedge x \notin F$ whenever $w \in F$ and $j_{2} \leq w \leq d_{2}$. Summing up, we have established $F^{\prime} \backslash F=\left\{w \wedge x: j_{2} \leq w \leq d_{2}\right\}$.

Finally, assume $w_{1}, w_{2} \in F, j_{2} \leq w_{1}, w_{2} \leq d_{2}$, and $w_{1} \wedge x=w_{2} \wedge x$. This implies $w_{1} \wedge d_{0}=w_{1} \wedge x \wedge d_{1}=w_{2} \wedge x \wedge d_{1}=w_{2} \wedge d_{0}$. Working in $L_{F}$, we have $\widehat{d_{2}} \backslash \widehat{d_{0}}=\left\{j_{1}, j_{2}\right\}$, and we conclude, observing that $j_{1} \leq j_{2} \leq w_{1}, w_{2}$, that $\widehat{w_{1}}=\left(\widehat{w_{1}} \cap \widehat{d_{0}}\right) \cup\left\{j_{1}, j_{2}\right\}=\left(\widehat{w_{2}} \cap \widehat{d_{0}}\right) \cup\left\{j_{1}, j_{2}\right\}=\widehat{w_{2}}$, that is, $w_{1}=w_{2}$.

Define a map $h$ from the interval $\left[j_{2}, d_{2}\right] \subseteq F$ to $F^{\prime} \backslash F$ by $h(w)=w \wedge x$. So $h$ is onto and injective by the above, and clearly order-preserving. Assume $w \wedge x \leq w^{\prime} \wedge x$. Then $w \wedge w^{\prime} \wedge x=w \wedge x$, and thus $w \wedge w^{\prime}=w$ or $w \leq w^{\prime}$. So $h^{-1}$ also preserves order and the final result is that $h$ is an order-isomorphism between $\left[j_{2}, d_{2}\right] \subseteq F$ and $F^{\prime} \backslash F$.

Using $h$, we define a map $\phi: F^{\prime} \rightarrow B_{F}=\mathcal{P}(\mathcal{J}(F))$ by

$$
\phi(z)= \begin{cases}\hat{z} & \text { for } z \in F, \\ \hat{w} \backslash\left\{j_{1}\right\} & \text { for } z=h(w) \in F^{\prime} \backslash F .\end{cases}
$$


We want to determine $\phi\left[F^{\prime}\right] \subseteq B_{F}$. Since $\phi(z)=\mathrm{O}_{F}(z)$ for $z \in F$, we certainly have $L_{F} \subseteq \phi\left[F^{\prime}\right]$. So consider $z=h(w) \in F^{\prime} \backslash F$. Then $w \in\left[j_{2}, d_{2}\right] \subseteq F$, and thus $\hat{w}=\hat{u} \cup\left\{j_{1}, j_{2}\right\}$ for a uniquely determined $u \in\left[j_{2} \wedge d_{0}, d_{0}\right] \subseteq F$, namely $u=w \wedge d_{0}$. Consequently, $\phi(z)=\hat{u} \cup\left\{j_{2}\right\} \in B_{F} \backslash L_{F}$. Conversely, any set $\hat{u} \cup\left\{j_{2}\right\} \in B_{F}$ with $u \in\left[j_{2} \wedge d_{0}, d_{0}\right]$ has a unique preimage under $\phi$, given by $z=w \wedge x$ where $\hat{w}=\hat{u} \cup\left\{j_{1}, j_{2}\right\}$. Since $h$ is an order isomorphism, $\phi$ restricted to $F^{\prime} \backslash F$ thus provides an order isomorphism between $F^{\prime} \backslash F$ and $\left\{\hat{u} \cup\left\{j_{2}\right\}: u \in\left[j_{2} \wedge d_{0}, d_{0}\right]\right\}$ (the latter ordered by set inclusion).

Putting this all together, we see-where $\cong$ is an order isomorphism-

$$
F^{\prime} \cong L_{F} \cup\left\{U \cup\left\{j_{2}\right\}: U \in \mathcal{O}(\mathcal{J}(F)) \text { and } \downarrow j_{2} \cap H_{0} \subseteq U \subseteq H_{0}\right\} .
$$

By Lemma 5.2, the latter is just $\mathcal{O}\left(\left(\mathcal{J}(F)_{j_{1} j_{2}}^{\prime}\right)\right.$, a (distributive) down-set lattice. An order isomorphism between lattices is always a lattice isomorphism, so $F^{\prime} \cong \mathcal{O}\left(\mathcal{J}(F)_{j_{1} j_{2}}^{\prime}\right)$ as lattices and $\mathcal{J}\left(F^{\prime}\right) \cong \mathcal{J}(F)_{j_{1} j_{2}}^{\prime}$ as posets.

Corollary 5.4. Assume $F_{0}$ is a finite distributive p-subsemilattice of a distributive p-semilattice $P$ satisfying (A1). Then there exists a finite distributive p-semilattice $F_{1}$ such that $F_{0} \unlhd F_{1} \unlhd P$ and the dense filter $\mathrm{D}\left(F_{1}\right)$ is boolean.

Proof. Let $G_{0}=F_{0}$ and for $i \geq 0$, obtain $G_{i+1}$ from $G_{i}$ by applying Lemma 5.3 w.r.t. a covering pair of nonmimimal dense elements in $G_{i}$. The process stops when no such pair can be found; the final $G_{i_{0}}$ has a boolean dense filter by Lemma 5.1. Put $F_{1}=G_{i_{0}}$.

\section{Adding "central" elements to the skeleton}

In this section, we assume that $P$ is an arbitrary distributive p-semilattice, and $F \unlhd P$ a finite distributive non-boolean p-subsemilattice whose dense filter $\mathrm{D}(F)$ is boolean, that is, $\mathrm{D}(F) \cong \mathbf{2}^{n}$ for some $n \geq 1$. It follows that $\mathrm{D}(F)$ contains $n$ different maximally dense elements. Let $\mathrm{D}_{\max }(F)=\left\{d_{1}, \ldots, d_{n}\right\}$.

Our purpose is to show that there exists a finite distributive p-subsemilattice $F^{\prime} \unlhd P$ such that $F \unlhd F^{\prime} \unlhd P$ and $F^{\prime} \cong \mathbf{2}^{r} \times \prod_{i=1}^{n} \hat{B}_{i}$ for some $r \in \omega$ and $B_{i}$ a boolean algebra for $1 \leq i \leq n$, provided $P$ satisfies the following property (A2) of p-semilattices:

$$
\begin{gathered}
\left(\forall a \in \mathrm{Sk}(P), d, d^{\prime} \in \mathrm{D}(P), p, p^{\prime}, x \in P\right)(\exists z \in \mathrm{Sk}(P)) \\
\left(\left(d \| d^{\prime} \& p \leq d^{\prime} \& p^{\prime} \leq d \& p^{\prime} \not \leq d^{\prime} \& a \leq d \& a^{*} \wedge p \not \leq d \& x^{*} \leq d^{\prime}\right) \quad(\right. \\
\left.\Longrightarrow\left(a \leq z \leq d \& z^{*} \wedge p \not \leq d \& z \wedge p^{\prime} \leq d^{\prime} \&(z \wedge x)^{*} \leq d^{\prime}\right)\right) .
\end{gathered}
$$

Roughly speaking, boolean elements as provided by (A2) will be used to manufacture a finite extension $F^{\prime}$ of $F$ containing a decomposition $\left\{u_{1}, \ldots, u_{t}\right\}$ of $1_{F^{\prime}}=1_{F}$ into finitely many pairwise disjoint boolean elements such that $F^{\prime} / \theta_{u_{i}}$ is isomorphic to either 2 or $\hat{B}_{i}$ for $1 \leq i \leq t$.

We start by constructing, for every $d_{i} \in \mathrm{D}_{\max }(F)$, an element $k_{i} \in \operatorname{Sk}(P)$ satisfying certain properties, using (A2). This is accomplished in several steps. 
Observe first that the set $H_{i}:=\left\{x \in F: x \not \leq d_{i}\right\}$ is closed under meets for every $d_{i} \in \mathrm{D}_{\max }(F)$. Indeed, assume towards a contradiction that $x, x^{\prime} \in H_{i}$ but $x \wedge x^{\prime} \leq d_{i}$. By distributivity, we find $y, y^{\prime} \in F$ such that $y \geq x, y^{\prime} \geq x^{\prime}$, and $y \wedge y^{\prime}=d_{i}$. So, $y, y^{\prime} \in \mathrm{D}(F)$, and by maximality of $d_{i}$, we have $y=d_{i}$ or $y^{\prime}=d_{i}$. Without loss of generality, assume $y=d_{i}$. But this violates $x \not \leq d_{i}$, proving our claim.

For $1 \leq i \leq n$, define $m_{i}=\bigwedge H_{i}$; it follows that $m_{i}$ is the smallest element of $F$ not below $d_{i}$. Observe further that $m_{i} \leq d_{j}$ for any $j \neq i, 1 \leq j \leq n$ : we have $d_{j} \leq \leq d_{i}$ by the maximality of $d_{j}$, hence $m_{i} \leq d_{j}$ by the minimality of $m_{i}$.

These properties of the $d_{i}$ and $m_{i}$ together with (A2) prove the following lemma.

Lemma 6.1. Assume (A2) and suppose that $k \in \operatorname{Sk}(P)$ and $d_{i} \in \mathrm{D}_{\max }(F)$ satisfy $k \leq d_{i}$ and $k^{*} \wedge m_{i} \not \leq d_{i}$. Then for any $d_{j} \in \mathrm{D}_{\max }(F)$ with $j \neq i$, there exists $z \in \operatorname{Sk}(P)$ (depending on $j$ ) such that $k \leq z \leq d_{i}, z^{*} \wedge m_{i} \not \leq d_{i}$, $z \wedge m_{j} \not \leq d_{j}$, and satisfying that for all $x \in F, x^{*} \leq d_{j} \Rightarrow(z \wedge x)^{*} \leq d_{j}$.

Proof. Let $X_{j}=\left\{x \in F: x^{*} \leq d_{j}\right\}=\left\{x_{j 1}, \ldots, x_{j n(j)}\right\}$. Use (A2) with $a:=k$, $d:=d_{i}, d^{\prime}:=d_{j}, p:=m_{i}, p^{\prime}:=m_{j}$, and $x:=x_{j 1}$. (It is routine to check that the assumptions in (A2) are all satisfied). So, by (A2), there exists $z_{1} \in \operatorname{Sk}(P)$ such that $k \leq z_{1} \leq d_{i}, z_{1}^{*} \wedge m_{i} \not \leq d_{i}, z_{1} \wedge m_{j} \not \leq d_{j}$, and $\left(z_{1} \wedge x_{j 1}\right)^{*} \leq d_{j}$.

Now apply (A2) with $d, d^{\prime}, p, p^{\prime}$ as above but with $a:=z_{1}$ and $x:=x_{j 2}$ (again, all the assumptions in (A2) are satisfied). So we find $z_{2} \in \operatorname{Sk}(P)$ such that $z_{1} \leq z_{2} \leq d_{i}, z_{2}^{*} \wedge m_{i} \leq \leq d_{i}, z_{2} \wedge m_{j} \not \leq d_{j}$, and $\left(z_{2} \wedge x_{j 2}\right)^{*} \leq d_{j}$. Note that $z_{1} \leq z_{2}$ implies $z_{1} \wedge x_{j 1} \leq z_{2} \wedge x_{j 1}$, and thus $\left(z_{2} \wedge x_{j 1}\right)^{*} \leq\left(z_{1} \wedge x_{j 1}\right)^{*} \leq d_{j}$; consequently, $z_{2}$ also satisfies $\left(z_{2} \wedge x_{j 1}\right)^{*} \leq d_{j}$.

Continue until $X_{j}$ is exhausted. The final $z_{n(j)}$ has all of the properties required by the lemma, so put $z=z_{n(j)}$.

Lemma 6.2. Assume (A2). Then for every $d_{i} \in D_{\max }$, there exists $k_{i} \in$ $\operatorname{Sk}(P)$ such that $k_{i} \leq d_{i}, k_{i}^{*} \wedge m_{i} \not \leq d_{i}, k_{i} \wedge m_{j} \not \leq d_{j}($ for all $j \neq i)$, and satisfying that for all $x \in F, x^{*} \leq d_{j} \Rightarrow\left(k_{i} \wedge x\right)^{*} \leq d_{j}($ for all $j \neq i)$.

Proof. Assume, without loss of generality, that $i=1$, and put $h_{1}=0$. This means that $h_{1} \in \operatorname{Sk}(P), h_{1} \leq d_{1}$ and $h_{1}^{*} \wedge m_{1} \not \leq d_{1}$. Put $j=2$ and apply Lemma 6.1 in order to obtain an element $z$ satisfying $0=h_{1} \leq z \leq d_{1}$, $z^{*} \wedge m_{1} \not \leq d_{1}, z \wedge m_{2} \not \leq d_{2}$ and $\forall x \in F\left(x^{*} \leq d_{2} \Rightarrow(z \wedge x)^{*} \leq d_{2}\right)$. We put $h_{2}:=z$.

Put $j=3$ and repeat to obtain $h_{3} \in \operatorname{Sk}(P)$ satisfying $h_{2} \leq h_{3} \leq d_{1}$, $h_{3}^{*} \wedge m_{1} \not \leq d_{1}, h_{3} \wedge m_{3} \not \leq d_{3}$, and for all $x \in F, x^{*} \leq d_{3} \Rightarrow\left(h_{3} \wedge x\right)^{*} \leq d_{3}$.

Now $h_{3}$ works also for $d_{2}$ : indeed, since $h_{2} \leq h_{3}$, we have $h_{2} \wedge m_{2} \leq h_{3} \wedge m_{2}$, and so $h_{3} \wedge m_{2} \not \leq d_{2}$, since $h_{2} \wedge m_{2} \not \leq d_{2}$. Similarly, for any $x^{*} \leq d_{2}$, we have $h_{2} \wedge x \leq h_{3} \wedge x$, and so $\left(h_{3} \wedge x\right)^{*} \leq\left(h_{2} \wedge x\right)^{*} \leq d_{2}$.

Continuing, we finally obtain $h_{n} \in \operatorname{Sk}(P)$ satisfying $h_{n} \leq d_{1}, h_{n}^{*} \wedge m_{1} \not \leq d_{1}$, $h_{n} \wedge m_{j} \not \leq d_{j}$ (for all $j \neq 1$ ), and for all $x \in F, x^{*} \leq d_{j} \Rightarrow\left(h_{n} \wedge x\right)^{*} \leq d_{j}$ ) (for all $j \neq 1$ ). Put $k_{1}=h_{n}$. 
Note that $k_{i}^{*} \wedge m_{i} \not \leq d_{i}$ is equivalent to $k_{i}^{*} \wedge z \not \leq d_{i}$ for all $z \in H_{i}$. One direction is clear, since $m_{i} \in F$ and $m_{i} \not \leq d_{i}$. For the other, assume $z \in F$ and $z \not \leq d_{i}$. Then $z \geq m_{i}$ by definition of $m_{i}$, and thus $k_{i}^{*} \wedge z \geq k_{i}^{*} \wedge m_{i}$. So if $k_{i}^{*} \wedge m_{i} \not \leq d_{i}$, then $k_{i}^{*} \wedge z \not \leq d_{i}$ a fortiori. The same argument shows that $k_{i} \wedge m_{j} \not \leq d_{j}(j \neq i)$ is equivalent to $k_{i} \wedge z \not \leq d_{j}$ for all $z \in H_{j}$.

This gives the final description of the elements $k_{i} \in \operatorname{Sk}(P)$ we are after.

Lemma 6.3. For each element $d_{i} \in \mathrm{D}_{\max (F)}$, there exists $k_{i} \in \operatorname{Sk}(P)$ such that

(i) $k_{i} \leq d_{i}$,

(ii) for $z \in F, z \not \leq d_{i} \Rightarrow k_{i}^{*} \wedge z \not \leq d_{i}$,

(iii) for $j \neq i$ and $z \in F, z \not \leq d_{j} \Rightarrow k_{i} \wedge z \not \leq d_{j}$,

(iv) for $j \neq i$ and $x \in F, x^{*} \leq d_{j} \Rightarrow\left(k_{i} \wedge x\right)^{*} \leq d_{j}$.

For easier reference, we list some consequences of the preceding lemma.

Corollary 6.4. The elements $k_{i}$ described in Lemma 6.3 have the following additional properties:

(ii-bis) for $z \in F, k_{i}^{*} \wedge z \leq d_{i} \Rightarrow z \leq d_{i}$,

(iii-bis) for $j \neq i$ and $z \in F, k_{i} \wedge z \leq d_{j} \Rightarrow z \leq d_{j}$,

(v) for $y \in \operatorname{Sk}(F), y \leq d_{i} \Rightarrow k_{i} \sqcup y \leq d_{i}$,

(vi) for $y \in \operatorname{Sk}(F), y \leq d_{j} \Rightarrow k_{i}^{*} \sqcup y \leq d_{j}$.

Proof. (ii-bis) and (iii-bis) are just the contrapositions of (ii) and (iii), respectively, in the preceding lemma.

$(\mathrm{v}):$ Assume $y \in \operatorname{Sk}(F), y \leq d_{i}$, and put $z=k_{i} \sqcup y$. Then $k_{i}^{*} \wedge z=$ $k_{i}^{*} \wedge\left(k_{i} \sqcup y\right)=k_{i}^{*} \wedge y \leq y \leq d_{i}$. Using (ii-bis), we obtain $z \leq d_{i}$ as desired.

(vi): Assume $y \in \operatorname{Sk}(F), y \leq d_{j}$, and put $z=k_{i}^{*} \sqcup y$. Then $k_{i} \wedge z=$ $k_{i} \wedge\left(k_{i}^{*} \sqcup y\right)=k_{i} \wedge y \leq y \leq d_{j}$, which implies $z \leq d_{j}$, using (iii-bis).

Next, consider $F\left[k_{i}\right]$, the p-semilattice generated in $P$ by $F \cup\left\{k_{i}\right\}$. Write $\operatorname{Sk}(F)\left[k_{i}\right]$ for the (boolean) subalgebra of $\operatorname{Sk}(P)$ generated by $\operatorname{Sk}(F) \cup\left\{k_{i}\right\}$; it is easy to see that $\operatorname{Sk}\left(F\left[k_{i}\right]\right)=\operatorname{Sk}(F)\left[k_{i}\right]$. Moreover, $\mathrm{D}\left(F\left[k_{i}\right]\right)=\mathrm{D}(F)$. Since $F$ is distributive, and thus representable by Lemma 4.4, it follows that also $F\left[k_{i}\right]$ is representable.

Lemma 6.5. $F\left[k_{i}\right]$ is distributive.

Proof. Using Lemma 4.5, it suffices to show that each $d \in \mathrm{D}\left(F\left[k_{i}\right]\right)=\mathrm{D}(F)$ is distributive. But $\mathrm{D}(F)$ is boolean and finite, so every $d \in \mathrm{D}(F)$ is the meet of all $d_{j} \in \mathrm{D}_{\max }(F)$ covering $d$. Since the meet of distributive elements is always distributive, it remains to check that every $d_{j} \in \mathrm{D}_{\max }(F)$ is distributive in $F\left[k_{i}\right]$.

We have $F\left[k_{i}\right]=\left\{b \wedge d: b \in \operatorname{Sk}\left(F\left[k_{i}\right]\right), d \in \mathrm{D}\left(F\left[k_{i}\right]\right)\right\}$, since $F\left[k_{i}\right]$ is representable. Using conjunctive normal form for boolean terms and $\mathrm{D}\left(F\left[k_{i}\right]\right)=$ $\mathrm{D}(F)$, this boils down to

$$
F\left[k_{i}\right]=\left\{\left(a \sqcup k_{i}\right) \wedge\left(b \sqcup k_{i}^{*}\right) \wedge d: a, b \in \operatorname{Sk}(F), d \in \mathrm{D}(F)\right\} .
$$


So assume $d_{j} \geq v \wedge w$ with $d_{j} \in \mathrm{D}_{\max }(F)$ and $v, w \in F\left[k_{i}\right]$. We want to find $v^{\prime}, w^{\prime} \in F\left[k_{i}\right]$ such that $v^{\prime} \geq v, w^{\prime} \geq w$, and $v^{\prime} \wedge w^{\prime}=d_{j}$. Explicitly, $v$, respectively, $w$ is given as $v=\left(v_{1} \sqcup k_{i}\right) \wedge\left(v_{2} \sqcup k_{i}^{*}\right) \wedge d_{v}$, respectively, $w=\left(w_{1} \sqcup k_{i}\right) \wedge\left(w_{2} \sqcup k_{i}^{*}\right) \wedge d_{w}$ with $v_{1}, v_{2}, w_{1}, w_{2} \in \operatorname{Sk}(F)$ and $d_{v}, d_{w} \in \mathrm{D}(F)$.

Case 1: $j \neq i$. We have

$$
\begin{aligned}
d_{j} & \geq\left(\left(v_{1} \sqcup k_{i}\right) \wedge\left(v_{2} \sqcup k_{i}^{*}\right) \wedge d_{v}\right) \wedge\left(\left(w_{1} \sqcup k_{i}\right) \wedge\left(w_{2} \sqcup k_{i}^{*}\right) \wedge d_{w}\right) \\
& =\left(\left(v_{1} \wedge w_{1}\right) \sqcup k_{i}\right) \wedge\left(\left(v_{2} \wedge w_{2}\right) \sqcup k_{i}^{*}\right) \wedge d_{v} \wedge d_{w} \geq k_{i} \wedge\left(v_{2} \wedge w_{2}\right) \wedge d_{v} \wedge d_{w} .
\end{aligned}
$$

Putting $z=\left(v_{2} \wedge w_{2}\right) \wedge d_{v} \wedge d_{w}$, we have $d_{j} \geq k_{i} \wedge z$. Observe that $z \in F$; so Corollary 6.4(iii-bis) applies and gives $z \leq d_{j}$, that is, $d_{j} \geq\left(v_{2} \wedge w_{2}\right) \wedge\left(d_{v} \wedge d_{w}\right)$. Using distributivity of $F$, we find $d_{1} \geq v_{2} \wedge w_{2}, d_{2} \geq d_{v} \wedge d_{w}$ such that $d_{1} \wedge d_{2}=d_{j}$. This makes $d_{1}, d_{2}$ dense and thus $d_{1}=d_{j}$ or $d_{2}=d_{j}$, since $d_{j} \in \mathrm{D}_{\max }(F)$.

Suppose $d_{j}=d_{2}$. Then $d_{j} \geq d_{v} \wedge d_{w}$, and by distributivity of $F$ again, we find $d_{v}^{\prime} \geq d_{v}, d_{w}^{\prime} \geq d_{w}$ such that $d_{v}^{\prime} \wedge d_{w}^{\prime}=d_{j}$. By maximality of $d_{j}$, we must have $d_{v}^{\prime}=d_{j}$ or $d_{w}^{\prime}=d_{j}$. In the first case, we obtain $d_{j}=d_{v}^{\prime} \geq d_{v} \geq v$. Putting $v^{\prime}=d_{j}, w^{\prime}=1$, we realize $v^{\prime} \geq v, w^{\prime} \geq w$, and $v^{\prime} \wedge w^{\prime}=d_{j}$, as desired. If $d_{w}^{\prime}=d_{j}$, the analogous argument shows that $v^{\prime}=1$ and $w^{\prime}=d_{j}$ work as well.

It remains to consider the case $d_{j}=d_{1}$. This time, we have $d_{j} \geq v_{2} \wedge w_{2}$, and distributivity of $F$ provides $d_{v}^{\prime} \geq v_{2}, d_{w}^{\prime} \geq w_{2}$ such that $d_{v}^{\prime} \wedge d_{w}^{\prime}=d_{j}$. Again, $d_{j}=d_{v}^{\prime}$, and thus $d_{j} \geq v_{2}$, or $d_{j}=d_{w}^{\prime}$, and thus $d_{j} \geq w_{2}$.

Use Lemma 6.3(iv) with $x=1$ to obtain $d_{j} \geq k_{i}^{*}$. Applying Corollary 6.4(vi), we deduce that $d_{j} \geq v_{2} \sqcup k_{i}^{*}$ or $d_{j} \geq w_{2} \sqcup k_{i}^{*}$. In the first case, $d_{j} \geq\left(v_{2} \sqcup k_{i}^{*}\right) \wedge\left(v_{1} \sqcup k_{i}\right) \wedge d_{v}=v$; in the second, $d_{j} \geq\left(w_{2} \sqcup k_{i}^{*}\right) \wedge\left(w_{1} \sqcup k_{i}\right) \wedge d_{w}=v$. This shows that $v^{\prime}=d_{j}$ and $w^{\prime}=1$, respectively $v^{\prime}=1$ and $w^{\prime}=d_{j}$, have the desired properties.

Case 2: $j=i$. The arguments have the same structure as in the case $j \neq i$, so we give only an outline. Start from

$$
\begin{aligned}
d_{i} & \geq\left(\left(v_{1} \sqcup k_{i}\right) \wedge\left(v_{2} \sqcup k_{i}^{*}\right) \wedge d_{v}\right) \wedge\left(\left(w_{1} \sqcup k_{i}\right) \wedge\left(w_{2} \sqcup k_{i}^{*}\right) \wedge d_{w}\right) \\
& =\left(\left(v_{1} \wedge w_{1}\right) \sqcup k_{i}\right) \wedge\left(\left(v_{2} \wedge w_{2}\right) \sqcup k_{i}^{*}\right) \wedge d_{v} \wedge d_{w} \geq\left(v_{1} \wedge w_{1}\right) \wedge k_{i}^{*} \wedge d_{v} \wedge d_{w} .
\end{aligned}
$$

Put $z=\left(v_{1} \wedge w_{1}\right) \wedge d_{v} \wedge d_{w}$ to obtain $d_{i} \geq k_{i}^{*} \wedge z$. Since $z \in F$, Corollary 6.4(ii-bis) applies and gives $z \leq d_{i}$, that is, $d_{i} \geq\left(v_{1} \wedge w_{1}\right) \wedge\left(d_{v} \wedge d_{w}\right)$. By distributivity of $F$, find $d_{1} \geq v_{1} \wedge w_{1}, d_{2} \geq d_{v} \wedge d_{w}$ such that $d_{1} \wedge d_{2}=d_{i}$, thus $d_{1}=d_{i}$ or $d_{2}=d_{i}$.

If $d_{i}=d_{1}$, obtain $v^{\prime}=d_{i}$ and $w^{\prime}=1$, respectively $v^{\prime}=1$ and $w^{\prime}=d_{i}$, as in Case 1. If $d_{i}=d_{2}$, the same arguments work, using Lemma 6.3(i) and Corollary 6.4(v).

We are now ready to construct $F^{\prime} \unlhd P$ such that we have $F \unlhd F^{\prime} \unlhd P$ and $F^{\prime} \cong \mathbf{2}^{r} \times \prod_{i=1}^{n} \hat{B}_{i}$. Observe that $F\left[k_{i}\right]$ is a finite distributive p-subsemilattice of $P$ containing $F$ and having the same dense filter as $F$. So we can iterate the construction of $F\left[k_{i}\right]$ with some other $d_{i^{\prime}} \in \mathrm{D}_{\max }(F)$, finding an element 
$k_{i^{\prime}} \in \operatorname{Sk}(P)$ that has all the required properties with respect to $F\left[k_{i}\right]$ and thus, a fortiori, with respect to $F$.

Explicitly, let $G_{0}=F$ and for $1 \leq i \leq n$, put $G_{i}=G_{i-1}\left[k_{i}\right]$. Then define $F^{\prime}=G_{n} . \quad F^{\prime}$ is distributive, $\mathrm{D}\left(F^{\prime}\right)=\mathrm{D}(F)$, and in particular, $F^{\prime}$ has the following properties: for every element $d_{i} \in \mathrm{D}_{\max }\left(F^{\prime}\right)$, there is $k_{i} \in \operatorname{Sk}\left(F^{\prime}\right)$ with

(i') $k_{i} \leq d_{i}$ by $6.3(\mathrm{i})$,

(ii') $k_{i}^{*} \not \leq d_{i}$ by 6.3 (ii), setting $z=1$,

(iii') $k_{i} \not \leq d_{j}$ by 6.3 (iii) for $j \neq i$, setting $z=1$,

$\left(\mathrm{iv}^{\prime}\right) k_{i}^{*} \leq d_{j}$ by $6.3(\mathrm{iv})$ for $j \neq i$, setting $x=1$.

Proposition 6.6. For some $r \in \omega, F^{\prime} \cong \mathbf{2}^{r} \times \prod_{i=1}^{n} \hat{B}_{i}$, with $B_{i}$ a boolean algebra for $1 \leq i \leq n$.

Proof. Let $C=\left\{a \in \mathrm{Sk}\left(F^{\prime}\right): a \leq d \in \mathrm{D}\left(F^{\prime}\right)\right.$ implies $\left.d=1\right\} . \quad C \neq \emptyset$, since $1 \in C$. Moreover, $C$ is closed under meets. Let $a, b \in C$ and $a \wedge b \leq d \in \mathrm{D}\left(F^{\prime}\right)$. By distributivity, we find $d_{a}, d_{b}$ such that $d_{a} \geq a, d_{b} \geq b$, and $d_{a} \wedge d_{b}=d$. Hence, $d_{a}, d_{b} \in \mathrm{D}\left(F^{\prime}\right)$, and so $d_{a}=d_{b}=1$, thus $d=1$. Since $F^{\prime}$ is finite, $c_{0}:=\bigwedge C$ exists and is the smallest element of $C$. Note that $c_{0} \neq 0$ (otherwise $F^{\prime}$ would be boolean).

The elements of $F^{\prime}$ have a canonical form. For $b \in \operatorname{Sk}\left(F^{\prime}\right)$, let $\Delta_{b}=$ $\left\{d_{l} \in \mathrm{D}_{\max }: b \not \leq d_{l}\right\}$. Since $F^{\prime}$ is representable and $\mathrm{D}\left(F^{\prime}\right)$ is boolean, it is clear that for every $u \in F^{\prime}$, there exists a representation $u=b \wedge \wedge Q$ with $b \in \operatorname{Sk}\left(F^{\prime}\right)$ and some subset $Q \subseteq \Delta_{b}$ (note that $\bigwedge Q=1$ iff $Q=\emptyset$ ). Assume $u=b \wedge \bigwedge Q=b^{\prime} \wedge \bigwedge Q^{\prime}$ are two different representations of this type. Applying **, we obtain $u^{* *}=b=b^{\prime}$, so $Q \neq Q^{\prime}$, and we find, without loss of generality, an element $d \in Q \backslash Q^{\prime}$. Note that $Q^{\prime} \neq \emptyset$ for otherwise $\wedge Q^{\prime}=1$, and so $b=b \wedge 1=b \wedge \wedge Q$, implying $b \leq \wedge Q \leq d$. Writing $Q^{\prime}=\left\{d_{1}^{\prime}, \ldots, d_{t}^{\prime}\right\}$, we obtain $d \geq b \wedge d_{1}^{\prime} \wedge \cdots \wedge d_{t}^{\prime}$. By distributivity, there are $v, w \in F^{\prime}$ such that $v \geq b, w \geq d_{1}^{\prime} \wedge \cdots \wedge d_{t}^{\prime}$, and $v \wedge w=d$. By maximality of $d$, it follows that $v=d$ or $w=d$. But $v=d$ is not possible, since $d \nsucceq b$, so we must have $w=d$, that is, $d \geq d_{1}^{\prime} \wedge \cdots \wedge d_{t}^{\prime}$. This implies the existence of $y, z$ such that $y \geq d_{1}^{\prime}, z \geq d_{2}^{\prime} \wedge \cdots \wedge d_{t}^{\prime}$, and $y \wedge z=d$. Repeat the procedure, using distributivity successively, to obtain finally that $d=d_{s}^{\prime}$ for some $d_{s}^{\prime} \in Q^{\prime}$, which contradicts our choice of $d$. It follows that $Q=Q^{\prime}$. So there is a unique subset $Q_{u} \subseteq \Delta_{u^{* *}}$ such that $u=u^{* *} \wedge \wedge Q_{u}$, and obviously $Q_{u}=\left\{d_{l} \in \mathrm{D}_{\max }: u \leq d_{l}\right.$ and $\left.u^{* *} \not \leq d_{l}\right\}$. Consequently, the correspondence $u \longleftrightarrow\left(u^{* *}, Q_{u}\right)$ is bijective.

For $1 \leq i \leq n$, define $a_{i}=k_{i} \sqcup c_{0}^{*} \in \operatorname{Sk}\left(F^{\prime}\right)$. We claim that $a_{i} \sqcup a_{j}=1$ for $i \neq j$ : Let $a_{i} \sqcup a_{j} \leq d \in D\left(F^{\prime}\right)$. If $d \neq 1$, there exists $d_{k} \in \mathrm{D}_{\max }\left(F^{\prime}\right)$ such that $d \leq d_{k}$, implying $k_{i} \leq a_{i} \leq d_{k}$ and $k_{j} \leq a_{j} \leq d_{k}$. By (iii') above, we have $i=k$ and $j=k$, contradicting $i \neq j$. Thus, $d=1$ and, consequently, $a_{i} \sqcup a_{j} \geq c_{0}$ by the definition of $c_{0}$. But $a_{i}, a_{j} \geq c_{0}^{*}$ by the definition of the $a_{i}$, so $a_{i} \sqcup a_{j} \geq c_{0} \sqcup c_{0}^{*}=1$, as claimed. 
By definition, $a_{i}^{*}=\left(k_{i} \sqcup c_{0}^{*}\right)^{*}=k_{i}^{*} \wedge c_{0}$. Note that $a_{i}^{*} \neq 0$ : Otherwise, $k_{i}^{*} \wedge c_{0}=0$, which implies $c_{0} \leq k_{i}^{* *}=k_{i} \leq d_{i}$ (by (i') above), contradicting the definition of $c_{0}$. We have $a_{i}^{*} \wedge a_{j}^{*}=\left(a_{i}^{*} \wedge a_{j}^{*}\right)^{* *}=\left(a_{i} \sqcup a_{j}\right)^{*}=1^{*}=0$ for $i \neq j$. Moreover, $a_{i}^{*} \wedge c_{0}^{*}=k_{i}^{*} \wedge c_{0} \wedge c_{0}^{*}=0$ for $1 \leq i \leq n$.

On the other hand, $a_{1}^{*} \sqcup \cdots \sqcup a_{n}^{*}=\left(k_{1}^{*} \sqcup \cdots \sqcup k_{n}^{*}\right) \wedge c_{0} \leq c_{0}$. If we have that $\left(k_{1}^{*} \sqcup \cdots \sqcup k_{n}^{*}\right) \wedge c_{0}<c_{0}$, there exists $1 \neq d \in \mathrm{D}\left(F^{\prime}\right)$-and with that, $d_{l} \in \mathrm{D}_{\max }\left(F^{\prime}\right)$ - such that $\left(k_{1}^{*} \sqcup \cdots \sqcup k_{n}^{*}\right) \wedge c_{0} \leq d \leq d_{l}$. Using distributivity, we find $d_{1} \geq k_{1}^{*} \sqcup \cdots \sqcup k_{n}^{*}$ and $d_{2} \geq c_{0}$ such that $d_{1} \wedge d_{2}=d_{l}$. Hence, $d_{1}, d_{2} \in \mathrm{D}\left(F^{\prime}\right)$, and thus $d_{2}=1$, which gives $d_{1}=d_{l}$. But $d_{l} \geq k_{1}^{*} \sqcup \cdots \sqcup k_{n}^{*}$ implies $d_{l} \geq k_{l}^{*}$, contradicting (ii') above. Consequently, $a_{1}^{*} \sqcup \cdots \sqcup a_{n}^{*}=\left(k_{1}^{*} \sqcup \cdots \sqcup k_{n}^{*}\right) \wedge c_{0}=c_{0}$. Summing up, we see that $\left\{c_{0}^{*}, a_{1}^{*}, \ldots, a_{n}^{*}\right\}$ provides a boolean partition of 1 .

We next determine the structure of the factor algebras $F^{\prime} / \theta_{a_{i}^{*}}$ for $1 \leq i \leq n$. Now, $F^{\prime} / \theta_{a_{i}^{*}} \cong\left\{u \wedge a_{i}^{*}: u \in F^{\prime}\right\}$ (the latter with the operations given in Section 2). So we have to compute the meets $u^{* *} \wedge \wedge Q_{u} \wedge a_{i}^{*}$ for $u \in F^{\prime}$. Now, $a_{i}^{*}=k_{i}^{*} \wedge c_{0}$, and $k_{i}^{*} \leq d_{l}$ for $l \neq i$ by (iv') above, so $u \wedge a_{i}^{*}=u^{* *} \wedge a_{i}^{*}$ if $d_{i} \notin Q_{u}$, and $u \wedge a_{i}^{*}=u^{* *} \wedge a_{i}^{*} \wedge d_{i}$ if $d_{i} \in Q_{u}$. We distinguish the cases $u^{* *} \geq a_{i}^{*}$, respectively, $u^{* *} \nsucceq a_{i}^{*}$.

First, assume that $u^{* *} \geq a_{i}^{*}$. Then $u \wedge a_{i}^{*}=a_{i}^{*}$ if $d_{i} \notin Q_{u}$, and $u \wedge a_{i}^{*}=a_{i}^{*} \wedge d_{i}$ if $d_{i} \in Q_{u}$. We claim that $a_{i}^{*} \wedge d_{i}<a_{i}^{*}$. If not, $a_{i}^{*}=k_{i}^{*} \wedge c_{0} \leq d_{i}$. But then, by distributivity, there are $v, w$ such that $k_{i}^{*} \leq v, c_{0} \leq w$, and $v \wedge w=d_{i}$, implying $v=d_{i}$ or $w=d_{i}$. Now $w=d_{i}$ yields $c_{0} \leq d_{i}$, which is not possible, and $v=d_{i}$ means $k_{i}^{*} \leq d_{i}$, violating $\left(\mathrm{ii}^{\prime}\right)$. Thus, $a_{i}^{*} \wedge d_{i}<a_{i}^{*}$ as claimed. Since $\left(a_{i}^{*} \wedge d_{i}\right)^{* *}=a_{i}^{*}$, we see that $a_{i}^{*} \wedge d_{i} \notin \operatorname{Sk}\left(F^{\prime}\right)$.

Next, suppose $u^{* *} \nsupseteq a_{i}^{*}$. Then $u^{* *} \wedge a_{i}^{*}<a_{i}^{*}$. We have $\left(u^{* *} \wedge a_{i}^{*}\right) \sqcup$ $k_{i} \nsucceq c_{0}$. (Since meeting $\left(u^{* *} \wedge a_{i}^{*}\right) \sqcup k_{i} \geq c_{0}$ on both sides with $k_{i}^{*}$ gives $u^{* *} \wedge a_{i}^{*} \wedge k_{i}^{*} \geq k_{i}^{*} \wedge c_{0}=a_{i}^{*}$, violating $u^{* *} \wedge a_{i}^{*}<a_{i}^{*}$.) So there exists $d_{l}$ such that $d_{l} \geq\left(u^{* *} \wedge a_{i}^{*}\right) \sqcup k_{i}$, whence $d_{l} \geq k_{i}$, and thus $l=i$ by (iii'). We conclude that $d_{i} \geq\left(u^{* *} \wedge a_{i}^{*}\right) \sqcup k_{i} \geq u^{* *} \wedge a_{i}^{*}$. So $u^{* *} \wedge a_{i}^{*} \leq a_{i}^{*} \wedge d_{i}$ (in fact, $u^{* *} \wedge a_{i}^{*}<a_{i}^{*} \wedge d_{i}$, since $a_{i}^{*} \wedge d_{i}$ is non-boolean), and we obtain $u \wedge a_{i}^{*}=u^{* *} \wedge a_{i}^{*}$ whether $d_{i} \in Q_{u}$ or not.

In other words, $\left\{u \wedge a_{i}^{*}: u \in F^{\prime}\right\}$ consists of $a_{i}^{*}, a_{i}^{*} \wedge d_{i}$, and all $b^{\prime} \in \operatorname{Sk}\left(F^{\prime}\right)$ with $b^{\prime}<a_{i}^{*}$, and the latter all satisfy $b^{\prime} \leq a_{i}^{*} \wedge d_{i}$. Moreover, $a_{i}^{*} \wedge d_{i}$ is the only non-boolean element occurring. It follows that $F^{\prime} / \theta_{a_{i}^{*}} \cong \hat{B}_{i}$ with $B_{i}$ a finite boolean algebra.

It remains to compute $F^{\prime} / \theta_{c_{0}^{*}}$. Consider $d_{l} \in \mathrm{D}_{\max }\left(F^{\prime}\right)$. Clearly, $d_{l} \geq 0=$ $c_{0} \wedge c_{0}^{*}$, so there are $u, v$ such that $u \geq c_{0}, v \geq c_{0}^{*}$, and $u \wedge v=d_{l}$. This implies $u=1$ (since $u \in \mathrm{D}\left(F^{\prime}\right)$ and $c_{0} \in C$ ), thus $v=d_{l}$. This shows that $d_{l} \geq c_{0}^{*}$ for all $d_{l} \in \mathrm{D}_{\max }\left(F^{\prime}\right)$.

Again, $F^{\prime} / \theta_{c_{0}^{*}} \cong\left\{u \wedge c_{0}^{*}: u \in F^{\prime}\right\}$ (the latter with the operations given in Section 2). So we have to compute the meets $u^{* *} \wedge \wedge Q_{u} \wedge c_{0}^{*}$ for $u \in F^{\prime}$. But since $d_{l} \geq c_{0}^{*}$ for any $d_{l} \in \mathrm{D}_{\max }\left(F^{\prime}\right)$, so $u^{* *} \wedge \wedge Q_{u} \wedge c_{0}^{*}=u^{* *} \wedge c_{0}^{*}$, and so we have $u \wedge c_{0}^{*}=u^{* *} \wedge c_{0}^{*}$ for all $u \in F^{\prime}$, whence

$$
\left\{u \wedge c_{0}^{*}: u \in F^{\prime}\right\}=\left\{b \in \operatorname{Sk}\left(F^{\prime}\right): b \leq c_{0}^{*}\right\} .
$$


It follows that $F^{\prime} / \theta_{c_{0}^{*}}$ is a finite boolean algebra. Note that $F^{\prime} / \theta_{c_{0}^{*}}$ is the trivial one-element algebra if $c_{0}=1$.

Let the canonical homomorphism $h: F^{\prime} \rightarrow F^{\prime} / \theta_{c_{0}^{*}} \times \prod_{i=1}^{n} F^{\prime} / \theta_{a_{i}^{*}}$ be given by $h(u):=\left(u \wedge c_{0}^{*}, u \wedge a_{1}^{*}, \ldots, u \wedge a_{n}^{*}\right)$; it is injective and surjective.

For injectivity, consider $v, w \in F^{\prime}, v \neq w$. Suppose first that $v^{* *} \neq w^{* *}$. Since $\left\{c_{0}^{*}, a_{1}^{*}, \ldots, a_{n}^{*}\right\}$ is a partition, so $v^{* *} \wedge c_{0}^{*} \neq w^{* *} \wedge c_{0}^{*}$ or $v^{* *} \wedge a_{l}^{*} \neq w^{* *} \wedge a_{l}^{*}$ for some $1 \leq l \leq n$. In the first case, we are done, since $u \wedge c_{0}^{*}=u^{* *} \wedge c_{0}^{*}$ for all $u \in F^{\prime}$, and thus $v \wedge c_{0}^{*} \neq w \wedge c_{0}^{*}$. In the second, remember that $u \wedge a_{i}^{*}$ equals $a_{i}^{*}$ or $a_{i}^{*} \wedge d_{i}$ if $u^{* *} \geq a_{i}^{*}$, and $u^{* *} \wedge a_{i}^{*}$ if $u^{* *} \nsucceq a_{i}^{*}$ (and then $u^{* *} \wedge a_{i}^{*}<a_{i}^{*} \wedge d_{i}$ ). Since $v^{* *} \wedge a_{l}^{*} \neq w^{* *} \wedge a_{l}^{*}$, we cannot have $v^{* *}, w^{* *} \geq a_{l}^{*}$, so suppose, without loss of generality, that $v^{* *} \nsupseteq a_{l}^{*}$, which implies $v \wedge a_{l}^{*}=v^{* *} \wedge a_{l}^{*}$. If also $w^{* *} \nsucceq a_{l}^{*}$, then $w \wedge a_{l}^{*}=w^{* *} \wedge a_{l}^{*} \neq v^{* *} \wedge a_{l}^{*}=v \wedge a_{l}^{*}$, and we are done. If $w^{* *} \geq a_{l}^{*}$, then $v \wedge a_{l}^{*}=v^{* *} \wedge a_{l}^{*}<a_{l}^{*} \wedge d_{l} \leq w \wedge a_{l}^{*}$, settling also this case.

Now suppose $v^{* *}=w^{* *}$. This implies $Q_{v} \neq Q_{w}$, so assume, without loss of generality, that there is $d_{l} \in Q_{w} \backslash Q_{v}$. It follows that $v \leq d_{l}$ but $w \not \leq d_{l}$. We infer that $v \wedge a_{l}^{*} \leq a_{l}^{*} \wedge d_{l}$. Suppose, towards a contradiction, that $v \wedge a_{l}^{*}=w \wedge a_{l}^{*}$. Then $w \wedge a_{l}^{*} \leq d_{l}$ and, by distributivity, we find $x, y$ such that $x \geq v, y \geq a_{l}^{*}$ and $x \wedge y=d_{l}$. As usual, we must have $x=d_{l}$ or $y=d_{l}$, which forces the contradiction $v \leq d_{l}$, respectively, $a_{l}^{*} \leq d_{l}$. Thus, $v \wedge a_{l}^{*} \neq w \wedge a_{l}^{*}$, as desired.

For surjectivity, consider, without loss of generality,

$$
w=\left(b_{0}, b_{1}, \ldots, b_{k}, b_{k+1} \wedge d_{k+1}, \ldots, b_{n} \wedge d_{n}\right)
$$

in $F^{\prime} / \theta_{c_{0}^{*}} \times \prod_{i=1}^{n} F^{\prime} / \theta_{a_{i}^{*}}$ with $b_{0}, \ldots, b_{n} \in \operatorname{Sk}\left(F^{\prime}\right), b_{0} \leq c_{0}$, and $b_{j} \leq a_{j}^{*}$ for $1 \leq j \leq n$, and $d_{l} \in \mathrm{D}_{\max }\left(F^{\prime}\right)$ for $k+1 \leq l \leq n$. It follows that $w^{* *}=$ $\left(b_{0}, \ldots, b_{n}\right)$. Put $x=\left(b_{0} \sqcup \cdots \sqcup b_{n}\right) \wedge d_{k+1} \wedge \cdots \wedge d_{n}$. Then $h(x)=w$.

Corollary 6.7. Assume $P$ is an arbitrary distributive $p$-semilattice satisfying (A2), and $F_{1} \unlhd P$ a finite distributive $p$-semilattice such that $\mathrm{D}\left(F_{1}\right) \cong \mathbf{2}^{n}$ for some $n \geq 1$. Then there exists a finite distributive $p$-semilattice $F_{2}$ such that $F_{1} \unlhd F_{2} \unlhd P$ and $F_{2} \cong \mathbf{2}^{r} \times \prod_{i=1}^{n} \hat{B}_{i}$, for some $r \in \omega$ and $B_{i}$ a boolean algebra for $1 \leq i \leq n$.

Proof. Use $F_{2}:=F^{\prime}$ as provided by Proposition 6.6.

\section{Extending factors $\hat{F}_{n}$ to $\hat{\mathbf{A}}$}

Assume that $P$ is an arbitrary distributive p-semilattice, $F \unlhd P$ a finite distributive p-semilattice of the form $F \cong \mathbf{2}^{r} \times \prod_{i=1}^{s} \hat{B}_{i}$ for some $r \in \omega$, and $B_{i}$ a finite boolean algebra for $1 \leq i \leq s$. We will show that $F$ can be extended to a p-semilattice $F^{\prime}$ such that $F \unlhd F^{\prime} \unlhd P$ and $F^{\prime} \cong \mathbf{2}^{r} \times(\hat{\mathbf{A}})^{s}$ (with A the countable atom-free boolean algebra), provided $P$ satisfies the following property (A3):

$$
\begin{aligned}
\left(\forall b_{1} \in \operatorname{Sk}(P),\right. & d \in \mathrm{D}(P))\left(\exists b_{2} \in \operatorname{Sk}(P)\right) \\
& \left(b_{1}<d<1 \Longrightarrow b_{1}<b_{2}<d \& b_{1} \sqcup b_{2}^{*}<d\right) .
\end{aligned}
$$


The key ingredient needed to prove the above statement is contained in the following lemma.

Lemma 7.1. Let $F \cong \hat{F}_{k} \times F^{\prime}$, with $k \geq 1$ and $F^{\prime}$ any finite distributive p-semilattice. If $P$ is any distributive $p$-semilattice satisfying (A3) and $F \unlhd P$, there exists $F^{+} \cong \hat{F}_{k+1} \times F^{\prime}$ such that $F \unlhd F^{+} \unlhd P$. Such $F^{+}$can be obtained by "splitting" any atom of $\hat{F}_{k}$.

Proof. Assume $F \cong \hat{F}_{k} \times F^{\prime} \unlhd P$ and $k \geq 2$. Let $c=(1,0)$ and $c^{*}=(0,1)$ be the central elements of $F$ associated with the direct product decomposition of $F$ specified above. Pick an atom of $F$ such that $a \leq c$. It follows that $a \in \mathrm{Sk}(F)$; moreover, $a^{*}$ is a coatom of $\mathrm{Sk}(F)$ and $a^{*} \geq c^{*}$. Further, let $e \in \mathrm{D}(F)$ be the unique dense element satisfying $e \neq 1$ and $e \geq c^{*}$. Now use (A3) to find $u^{*} \in \operatorname{Sk}(P)$ such that $a^{*}<u^{*}<e$ and $a^{*} \sqcup u<e$.

We have $u<a$, since $u^{*}>a^{*}$, and $u \neq 0$ (for otherwise $u^{*}=1 \not \leq e$ ), hence $0<u<a$. Consider $a \wedge u^{*}: a \wedge u^{*}=\left(a^{*} \sqcup u\right)^{*} \neq 0$ (for otherwise $\left.\left(a^{*} \sqcup u\right)^{* *}=a^{*} \sqcup u=1 \not \leq e\right)$, and $a \wedge u^{*} \neq u^{*}$ (for otherwise $u^{*} \leq a$, whence $a^{*} \leq u^{* *}=u<a$, and thus $a=1$ ). Summing up, we have $0<a \wedge u^{*}<u^{*}$ and obviously $u \wedge\left(a \wedge u^{*}\right)=0$ and $u \sqcup\left(a \wedge u^{*}\right)=a$. So, $u$ and $a \wedge u^{*}$ provide a proper splitting of the atom $a$.

Let $F[u]$ be the p-semilattice generated $F \cup\{u\}$ within $P$. It is clear that $F[u]$ is representable, being generated by $\operatorname{Sk}(F)[u]$ (the p-semilattice generated by $\operatorname{Sk}(F) \cup\{u\}$ within $\operatorname{Sk}(P))$ together with $\mathrm{D}(F)$, and that $\operatorname{Sk}(F[u])=$ $\operatorname{Sk}(F)[u]$. So we start by describing $\operatorname{Sk}(F)[u]$.

Note that every $x \in \operatorname{Sk}(F)$ has a unique representation $x=x_{1} \sqcup x_{2}$ with $x_{1} \leq c$ and $x_{2} \leq c^{*}$ : take $x_{1}=x \wedge c$ and $x_{2}=x \wedge c^{*}$. The same holds for $u$ with $u_{1}=u$ and $u_{2}=0$, and $u^{*}$ with $\left(u^{*}\right)_{1}=u^{*} \wedge c$ and $\left(u^{*}\right)_{2}=u^{*} \wedge c^{*}=c^{*}$. Define $S \subseteq \operatorname{Sk}(F)[u]$ by $s \in S$ iff $s=s_{1} \sqcup s_{2}$, where $s_{1}$ is $x_{1}$ or $x_{1} \sqcup u$ or $x_{1} \wedge u^{*}$ for some $x_{1} \in F$ with $x_{1} \leq c$, and where $s_{2}=x_{2}$ for some $x_{2} \in F$ with $x_{2} \leq c^{*}$. It is routine to see that $S$ is closed under $\wedge, \sqcup$, and ${ }^{*}$ by checking cases (this boils down to checking that $S_{1}=\left\{s_{1}: s \in S\right\}$ is closed under $\wedge$, $\sqcup$, and ' where $\left.s_{1}^{\prime}=s_{1}^{*} \wedge c\right)$. Moreover, $S$ contains $u$, so $S=\operatorname{Sk}(F)[u]$.

For any member of $\mathrm{D}\left(F^{\prime}\right)=\left\{\delta_{t}: t \in T\right\}$, define $d_{t} \in \mathrm{D}(F)$ to be the dense element associated with $\left(1, \delta_{t}\right)$ in the direct product decomposition of $F$. It follows that every $d \in \mathrm{D}(F)$ can be written as $d_{1} \wedge d_{2}$ with $d_{1} \in\{e, 1\}$ and $d_{2}=d_{t}$ for some $t \in T$. Finally, since $F[u]$ is representable, any $w \in F[u]$ can be written as $w=s \wedge d_{1} \wedge d_{2}$ with $s \in S$ and $d_{1}, d_{2}$ as specified just above.

We determine $F[u] / \theta_{c} \cong\{w \wedge c: w \in F[u]\}$ (the latter under the operations specified in Section 2). Now, $w \wedge c=\left(s_{1} \sqcup s_{2}\right) \wedge d_{1} \wedge d_{2} \wedge c$, which reduces to $s_{1} \wedge d_{1}$, since $s_{1} \leq c \leq d_{2}$ and $c \wedge s_{2}=0$. Let $Q$ be the set of all atoms of $\operatorname{Sk}(F)$ lying below $c$; then $a \in Q$ and $Q$ has $k$ elements. If $s_{1}<c$, then $s_{1}$ is the boolean join of a proper subset of $(Q \backslash\{a\}) \cup\left\{u, a \wedge u^{*}\right\}$ that contains $k+1$ elements, and we conclude that $\operatorname{Sk}\left(F[u] / \theta_{c}\right) \cong \mathbf{2}^{k+1}$. By construction, all such $s_{1}<c$ are below $e \wedge c$, which is thus the only non-boolean element in $F[u] / \theta_{c}$. It follows that $F[u] / \theta_{c} \cong \hat{F}_{k+1}$. 
Working analogously from $w \wedge c^{*}=\left(s_{1} \sqcup s_{2}\right) \wedge d_{1} \wedge d_{2} \wedge c^{*}$, which simplifies to $s_{2} \wedge d_{2}$ due to $s_{2} \leq c^{*} \leq d_{1}$ and $c^{*} \wedge s_{1}=0$, we obtain directly that $F[u] / \theta_{c^{*}} \cong F^{\prime}$.

Finally, consider the map $h: F[u] \rightarrow F[u] / \theta_{c} \times F[u] / \theta_{c^{*}}$, which we define by $\left.h\left(\left(s_{1} \sqcup s_{2}\right) \wedge d_{1} \wedge d_{2}\right)\right)=\left(s_{1} \wedge d_{1}, s_{2} \wedge d\right)$. Since the component maps of $h$ are the canonical projections of $F[u]$ onto $F[u] / \theta_{c}$, respectively, $F[u] / \theta_{c^{*}}, h$ is a homomorphism that is bijective by construction.

It remains to check the case $k=1$. Use (A3) to obtain $u^{*} \in \operatorname{Sk}(P)$ such that $c^{*}<u^{*}<e$ and $c^{*} \sqcup u<e$. Proceed analogously (but more simply) as above to show that $F[u] / \theta_{c} \cong \hat{F}_{2}$ and that $F[u] \cong \hat{F}_{2} \times F^{\prime}$.

Corollary 7.2. Let $P$ be an arbitrary distributive p-semilattice satisfying (A3), and $F_{2} \unlhd P$ a finite distributive nonboolean $p$-semilattice of the form $F_{2} \cong \mathbf{2}^{r} \times \prod_{i=1}^{n} \hat{B}_{i}$, with $r \in \omega$ and $B_{i}$ a finite boolean algebra for $1 \leq i \leq n$. Then there exists a p-semilattice $F_{3}$ such that $F_{2} \unlhd F_{3} \unlhd P$ and $F_{3} \cong \mathbf{2}^{r} \times(\hat{\mathbf{A}})^{n}$ (with $\mathbf{A}$ the countable atom-free boolean algebra).

Proof. Put $G_{0}=F_{2}$ and, for $m \in \omega$, obtain $G_{m+1}$ from $G_{m}$ by splitting every (boolean) atom in each of the $s$ factors of $G_{m}$ of type $\hat{B}_{i}$, using Lemma 7.1 repeatedly. Then let $F_{3}=\bigcup_{m \in \omega} G_{m}$.

\section{Necessity}

In this section, we show that the axioms (DIST), (A1), (A2), and (A3) are also necessary for a p-semilattice $P$ to be algebraically closed. This is done by extending any finite set $\left\{a_{1}, \ldots, a_{n}\right\}$ of elements of an a.c. p-semilattice $P$ satisfying the assumptions of such an axiom, to a p-subsemilattice $S \unlhd P$ isomorphic to a direct product $\mathbf{2}^{r} \times \widehat{A}^{s}, r, s \in \omega$. This is possible, since the class PCS is locally finite, which makes Theorem 3.1 applicable. It will be shown then that within $S$, any element whose existence is postulated by the axiom under consideration actually can be found.

We carry this out in detail for (A2) only, as the procedure is rather straightforward for (DIST), (A1), and (A3).

So assume that $a \in \mathrm{Sk}(P), d, d^{\prime} \in \mathrm{D}(P)$, and $p, p^{\prime}, x \in P$ satisfy all of the following: $d \| d^{\prime}, p \leq d^{\prime}, p^{\prime} \leq d, p^{\prime} \leq d^{\prime}, a \leq d, a^{*} \wedge p \not \leq d$, and $x^{*} \leq d^{\prime}$. Since $P$ is a.c., there is a p-sub-semilattice $S \unlhd P$ of $P$ isomorphic to a direct product $\mathbf{2}^{r} \times \widehat{A}^{s}$, for $r, s \in \omega$, containing $\left\{a, d, d^{\prime}, p, p^{\prime}, x\right\}$.

We will now define an element $z \in \operatorname{Sk}(S)$ satisfying $a \leq z \leq d, z^{*} \wedge p \not \leq d$, $z \wedge p^{\prime} \not \leq d^{\prime}$, and $(z \wedge x)^{*} \leq d^{\prime}$, by specifying its components $\left(z_{1}, \ldots, z_{r+s}\right)$. We distinguish four cases according to the values of $d_{i}$ and $d_{i}^{\prime}$ for $1 \leq i \leq r+s$.

(1) $d_{i}=d_{i}^{\prime}=1$ : Put $z_{i}=a_{i}$.

(2) $d_{i}=1, d_{i}^{\prime}=e:$ Put $z_{i}=1$.

(3) $d_{i}=e, d_{i}^{\prime}=1$ : Put $z_{i}=a_{i}$.

(4) $d_{i}=d_{i}^{\prime}=e$ 
(a) $a_{i} \wedge x_{i} \neq 0$ : Put $z_{i}=a_{i}$.

(b) $a_{i} \wedge x_{i}=0$ : This implies $a_{i} \leq x_{i}^{*}<d_{i}^{\prime}=e$ (remember $x^{*} \leq d^{\prime}$ ). Since $\pi_{i}[S]=\hat{\mathbf{A}}$, there is $b_{i} \in \pi_{i}[S]$ with $x_{i}^{*}<b_{i}<e$ (where $\pi_{i}$ stands for the projection of $S$ onto its $i$-th factor). Put $z_{i}=b_{i}$.

We claim that $z$ has all of the required properties:

- $a \leq z \leq d$ obviously is satisfied.

- $z^{*} \wedge p \not \leq d$ : Since $a^{*} \wedge p \not \leq d$ and $p \leq d^{\prime}$, there is $i \in\{r+1, \ldots, r+s\}$ such that $a_{i}^{*} \wedge p_{i} \not \leq d$, thus $a_{i}^{*}=p_{i}=1, d_{i}=e$, and $d_{i}^{\prime}=1$. This is Case 3 of the definition of $z$, hence $z_{i}=a_{i}$, and $\left(z^{*} \wedge p\right)_{i}=z_{i}^{*} \wedge p_{i}=a_{i}^{*} \wedge p_{i}=1 \not \leq e=d_{i}$, thus $z^{*} \wedge p \not \leq d$.

- $z \wedge p^{\prime} \not \leq d^{\prime}$ : Since $p^{\prime} \not \leq d^{\prime}$, there is $i \in\{r+1, \ldots, r+s\}$ such that $p_{i}^{\prime}=1$ and $d_{i}^{\prime}=e$. Because $p^{\prime} \leq d$, we have $d_{i}=1$. This is Case 2 of the definition of $z$, thus $z_{i}=1$. We obtain $\left(z \wedge p^{\prime}\right)_{i}=z_{i} \wedge p_{i}^{\prime}=1 \not \leq e=d_{i}^{\prime}$, thus $z \wedge p^{\prime} \not d^{\prime}$.

- $(z \wedge x)^{*} \leq d^{\prime}$ : We can assume $d_{i}^{\prime}=e$, since if $d_{i}^{\prime}=1$, we trivially have $\left(z_{x} \wedge x\right)_{i}^{*} \leq d_{i}^{\prime}$. So it remains to consider Cases 2, 4a, and $4 \mathrm{~b}$ of the definition of $z$.

In Case 2 , we have $z_{i}=1$, and so $(z \wedge x)_{i}^{*}=x_{i}^{*}$. But $x^{*} \leq d^{\prime}$ by assumption, thus $(z \wedge x)_{i}^{*} \leq d_{i}^{\prime}$.

In Case 4a, $a<d$ implies $0<a_{i} \wedge x_{i} \leq a_{i}<d_{i}=e$, and so also $0<\left(a_{i} \wedge x_{i}\right)^{*}<e$, and thus $(z \wedge x)_{i}^{*}<e=d_{i}^{\prime}$.

In Case $4 \mathrm{~b}$, we have $z_{i} \wedge x_{i} \leq z_{i}=b_{i}<e$. Moreover, $z_{i} \wedge x_{i} \neq 0$ (for otherwise $\left.z_{i}=b_{i} \leq x_{i}^{*}<b_{i}\right)$. It follows that $\left(z_{i} \wedge x_{i}\right)^{*}<e=d_{i}^{\prime}$.

\section{Main theorem}

The results obtained so far are enough to prove our main theorem:

Theorem 9.1. A p-semilattice $P$ is algebraically closed if and only if it is distributive and satisfies axioms (A1), (A2), and (A3).

Proof. Necessity is established in the preceding section. For sufficiency, let $F$ be a finite p-subsemilattice of a distributive p-semilattice $P$ satisfying (A1)(A3). By Corollary 4.11, $F$ can be extended within $P$ to a finite distributive p-subsemilattice $F_{0}$. By Corollary 5.4, $F_{0}$ can be extended within $P$ to a finite distributive p-subsemilattice $F_{1}$ whose dense filter is boolean. If $F_{1}$ is boolean, we are done, since then $F_{1} \cong \mathbf{2}^{r}$ and $s=0$. Otherwise, using Corollary 6.7, $F_{1}$ can be extended within $P$ to a p-subsemilattice $F_{2}$ such that $F_{2} \cong \mathbf{2}^{r} \times \prod_{i=1}^{n} \hat{B}_{i}$, for some $r \in \omega$ and $B_{i}$ a boolean algebra for $1 \leq i \leq n$. Finally, by Corollary $7.2, F_{2}$ can be extended within $P$ to a subsemilattice $F_{3}$ such that $F_{3} \cong$ $\mathbf{2}^{r} \times(\hat{\mathbf{A}})^{n}$ (with $\mathbf{A}$ the countable atom-free boolean algebra). 


\section{REFERENCES}

[1] Adler, J.: Model theoretic investigations of the class of pseudocomplemented semilattices. PhD thesis, University of Bern (1998)

[2] Adler, J.: The model companion of the class of pseudocomplemented semilattices is finitely axiomatizable (2012, preprint)

[3] Albert, M.H., Burris, S.N.: Finite axiomatizations for existentially closed posets and semilattices. Order 3, 169-178 (1986)

[4] Chaida, I., Halaš, R., Kühr, J.: Semilattice structures. Research and Exp. in Math. Vol. 30, Heldermann, Lemgo (2007)

[5] Frink, O.: Pseudo-Complements in Semi-Lattices. Duke Math. J. 29, 504-515 (1962)

[6] Hodges, W.: A shorter model theory. Cambridge Univ. Press, Cambridge (1997)

[7] Jones, G.-T.: Pseudocomplemented semilattices. PhD thesis, UCLA (1972)

[8] Katriňák, T.: Die Kennzeichnung der distributiven pseudokomplementären Halbverbände. J. Reine Angew. Math. 241, 160-179 (1970) (German)

[9] Pudlak, P.: On congruence lattices of lattices. Algebra Universalis 20, 96-114 (1985)

[10] Rupp, R.: On algebraically closed and existentially complete p-semilattices. PhD thesis, University of Bern (2006)

[11] Schmid, J.: Algebraically closed p-semilattices. Arch. Math. 45, 501-510 (1985)

JOËL ADLER

Gertrud-Woker-Strasse 5, Pädagogische Hochschule Bern, 3012 Bern, Switzerland e-mail: joel.adler@phbern.ch

\section{Regula Rupp}

Komturstrasse 34, 79106 Freiburg, Germany

e-mail: regularupp@gmail.com

\section{JÜRG SCHMID}

University of Bern, Mathematical Institute, Sidlerstrasse 5, 3012 Bern, Switzerland e-mail: juerg.schmid@math.unibe.ch 